\title{
Article \\ Crustaceans (Malacostraca and Thecostraca) from the International Minho River, Iberian Peninsula
}

\author{
Nuno Gomes ${ }^{1,2, *(\mathbb{D}}$, Dimítri A. Costa ${ }^{1,2} \mathbb{D}^{\mathbb{D}}$, Harold Cantallo ${ }^{1}$ and Carlos Antunes ${ }^{1,2} \mathbb{D}^{\mathbb{D}}$ \\ 1 Interdisciplinary Centre of Marine and Environmental Research (CIIMAR), University of Porto, Terminal de \\ Cruzeiros do Porto de Leixões, Avenida General Norton de Matos, s/n, 4450-208 Matosinhos, Portugal; \\ dimitri.costa@ciimar.up.pt (D.A.C.); haroldcantallo.hcc@gmail.com (H.C.); cantunes@ciimar.up.pt (C.A.) \\ 2 Aquamuseu do Rio Minho, Parque do Castelinho, 4920-290 Vila Nova de Cerveira, Portugal \\ * Correspondence: Nuno@ciimar.up.pt
}

\begin{abstract}
Crustaceans (Arthropoda) are a diverse and abundant group with chitinous exoskeleton, living on coastal/estuarine environments, at community invertebrate fauna. A survey on the species of some crustacean groups found on the Minho River estuary (international section) in the Iberian Peninsula, is presented with specimens collected through glass eel fishing bycatch, grab sampler, trammel net, beam trawl and fyke net sampling methods. A total of 98 specimens were examined belonging to 7 orders, 21 genera and 23 species (17 new records from Minho River, including one for Portugal). Brief diagnosis, ecological notes, species distributions and figures are provided intending to present taxonomic support on future projects in this region.
\end{abstract}

Keywords: Atlantic Ocean; distribution; estuary; invertebrates; taxonomy

check for

updates

Citation: Gomes, N.; Costa, D.A.; Cantallo, H.; Antunes, C. Crustaceans (Malacostraca and Thecostraca) from the International Minho River,

Iberian Peninsula. Hydrobiology 2022, 1, 47-75. https://doi.org/10.3390/ hydrobiology 1010005

Academic Editors: Lucian Pârvulescu and Octavian Pacioglu

Received: 29 October 2021

Accepted: 13 December 2021

Published: 22 December 2021

Publisher's Note: MDPI stays neutral with regard to jurisdictional claims in published maps and institutional affiliations.

Copyright: () 2021 by the authors Licensee MDPI, Basel, Switzerland. This article is an open access article distributed under the terms and conditions of the Creative Commons Attribution (CC BY) license (https:// creativecommons.org/licenses/by/ $4.0 /)$.

\section{Introduction}

The subphylum crustacea Brünnich, 1772 is one of the most numerous and diverse groups with more than 60,000 known species [1], with significant importance on estuarine ecosystems for providing a major source in fish and bird diets [2-5]. Additionally, these marine and brackish arthropods play various ecological roles, like predation [2], parasitism [6] and mesograzing [7]. They are widely used commercially (e.g., Crangon and Palaemon spp.) [2], for the food industry, pharmacological products, aquaculture and indirectly with research involving biotechnology for the reuse of waste from aquaculture [8]. Although crustaceans are a well-studied group, taxonomic problems arise from the lack of knowledge about certain groups, especially species with high sexual dimorphism or differences in juvenile-adult morphology $[2,9,10]$ compounded by information fragmentation. Estuarine ecosystems are of utmost biological importance, being productive and valuable for biodiversity maintenance [11]. Macroinvertebrate surveys on the Minho River estuary are still insufficient to access its biodiversity with few works on macrobenthic ecology or specimens collected through glass eel fishing bycatch [12-15] and a survey on crustaceans of the order Isopoda [16]. In this study we provide an overview of some groups of the Crustacean fauna (brief diagnosis, ecological notes, global and Portuguese distributions) collected on the Minho River estuary, with a total of 23 species, in which 17 are new records for the Minho River, including one new record for Portugal-Nebalia strausi Risso, 1826. In addition, an update to the list of isopods from Minho River is provided here.

\section{Materials and Methods}

\subsection{Study Area}

Located northwest of the Iberian Peninsula, the Minho River originates in the Meira mountains (Spain), with an extension of around $300 \mathrm{~km} \mathrm{[17].} \mathrm{The} \mathrm{last} \mathrm{section} \mathrm{of} 70 \mathrm{~km}$ represents the northwest Spanish/Portuguese border, which is part of the protected areas of the Natura 2000 network as a site of ecological importance and a special area of conservation, marked as an Important Bird Area $[18,19]$. The estuarine area has a total area of $23 \mathrm{~km}^{2}$ and 
a length of approximately $40 \mathrm{~km}$ [13], characterized by a mesotidal partially mixed system tending towards a salt wedge during the high floods [20].

\subsection{Sampling, Identification and Preservation of Specimens}

Specimens examined were collected during previous sampling campaign (including ecological researches from $[15,21]$ at the estuary of the international Minho River (Figure 1), with following methods: (1) glass eel fishing bycatch, in Caminha, Portugal $\left(41^{\circ} 52^{\prime} 59.00^{\prime \prime} \mathrm{N} / 8^{\circ} 50^{\prime} 14.00^{\prime \prime} \mathrm{W}\right)$, on April, 2020, on a new moon night during flood tides, using stow net (length of float lines $10 \mathrm{~m}$, bottom anchored lead line of $15 \mathrm{~m}$, height $8 \mathrm{~m}$, mesh size 1-2 mm, covering an area of $50 \mathrm{~m}^{2}$ ); (2) glass eel fishing bycatch, in Caminha, Portugal $\left(41^{\circ} 52^{\prime} 44.80^{\prime \prime} \mathrm{N} / 8^{\circ} 50^{\prime} 26.25^{\prime \prime} \mathrm{W}\right)$, on March 2021; (3) fyke nets (length $7 \mathrm{~m}$, mesh size $10 \mathrm{~mm}$, with two funnel shaped openings), in Vila Nova de Cerveira, Portugal ( $\left.41^{\circ} 57^{\prime} 1.69^{\prime \prime} \mathrm{N} / 8^{\circ} 44^{\prime} 42.74^{\prime \prime} \mathrm{W}\right)$; (4) on sediment sampling on saltmarsh with a Van Veen grab sampler in Caminha, Portugal ( $\left.41^{\circ} 52^{\prime} 30.42^{\prime \prime} \mathrm{N} / 8^{\circ} 49^{\prime} 52.97^{\prime \prime} \mathrm{W}\right)$ on March, 2006; (5) by hand on rock in Caminha, Portugal $\left(41^{\circ} 52^{\prime} 00^{\prime \prime} \mathrm{N} / 8^{\circ} 51^{\prime} 15.90^{\prime \prime} \mathrm{W}\right)$ on March 2021; (6) beam trawl in Caminha $\left(41^{\circ} 52^{\prime} 04.8^{\prime \prime} \mathrm{N} 8^{\circ} 51^{\prime} 18.8^{\prime \prime} \mathrm{W}\right.$ ) on June 2021; (7) in buccal cavity of Alosa alosa, captured with trammel fishing in Vila Nova de Cerveira, Portugal $\left(41^{\circ} 55^{\prime} 57.67^{\prime \prime} \mathrm{N} / 8^{\circ} 45^{\prime} 33.85^{\prime \prime} \mathrm{W}\right)$.
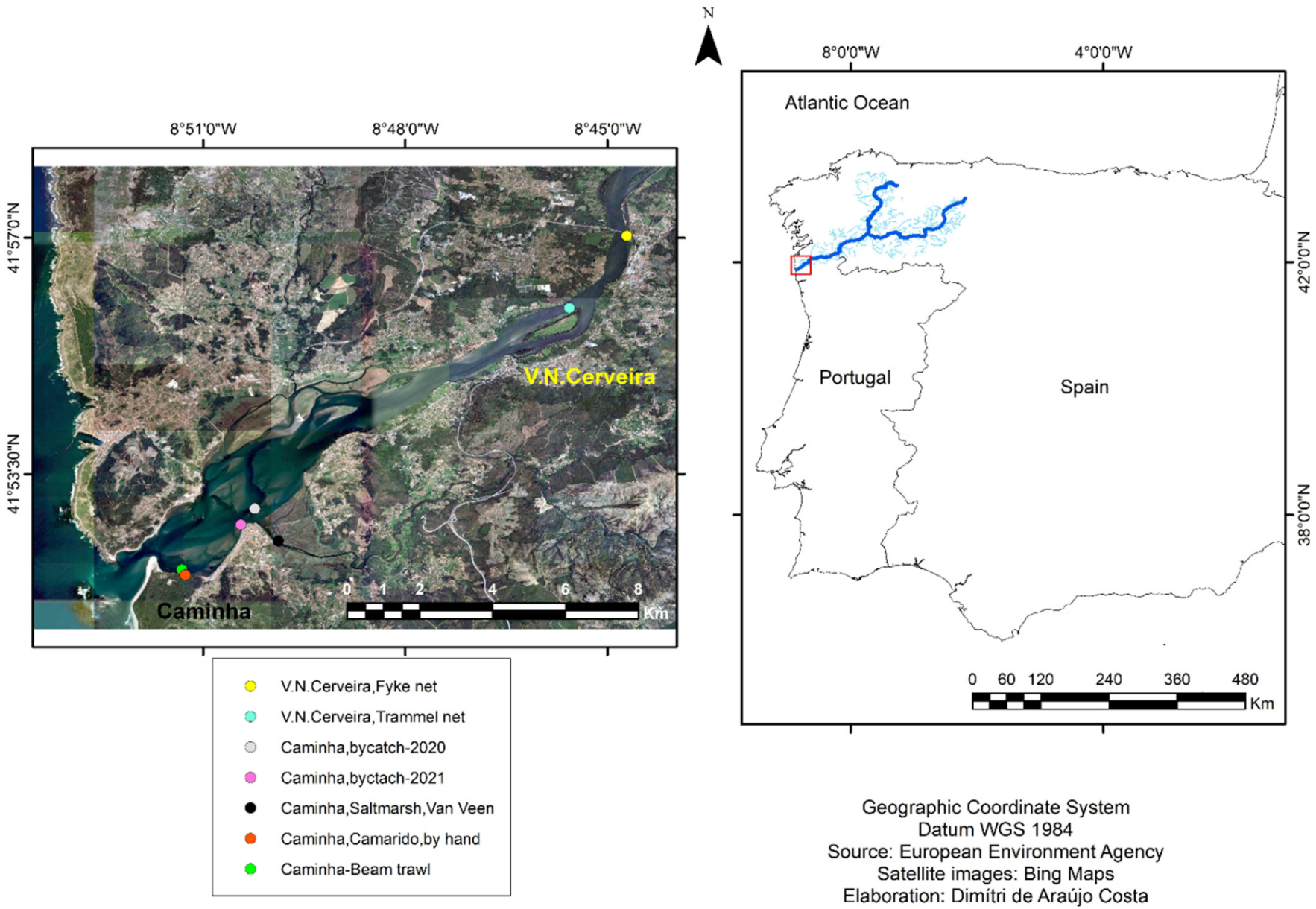

Figure 1. Study area of the international Minho River (northwest Iberian Peninsula), displaying the sampling points and methods. Satellite images adapted from Bing Maps.

Taxonomic identification was performed using specialized literature, e.g., [2,20-31]. Synonym compilation was based on WoRMS (World Register of Marine Species) database [32] and on species descriptions manuscripts. Original information data (material and locality type) data were compiled from GBIF (Global Biodiversity Information Facility) [33] or from 
first species descriptions, whenever available. Specimens were analyzed and photographed with a Nikon Digital Sight D5-L1 camera using a Nikon SMZ800 stereomicroscope and a Nikon ECLIPSE 50i microscope. All specimens were deposited at Arthropod Collection from Natural History Museum of the Iberian Peninsula (NatMIP_“Museu de História Natural da Península Ibérica"), at Aquamuseu do Rio Minho, Vila Nova de Cerveira, Portugal.

\subsection{DNA Extraction, Amplification and Sequencing}

DNA samples were extracted using E.Z.N.A. Mollusc DNA Kit (Omega Bio-tek). Amplification of the mitochondrial gene cytochrome c oxidase I (COI-5P), was performed using the primer pairs LoboF1 and LoboR1 [34]. PCR (Polymerase Chain Reaction) reactions contained $21 \mu \mathrm{L}$ of VWR company (Monroeville, PA, USA) Red Taq DNA Polymerase Master Mix, $1 \mu \mathrm{L}$ of each primer $(10 \mathrm{mM})$ and $2 \mu \mathrm{L}$ of DNA template. The PCR cycling conditions used were: $1 \mathrm{~min}$ at $94^{\circ} \mathrm{C} ; 5$ cycles of $30 \mathrm{~s}$ at $94{ }^{\circ} \mathrm{C}, 1.5 \mathrm{~min}$ at $45^{\circ} \mathrm{C}, 1 \mathrm{~min}$ at $72{ }^{\circ} \mathrm{C}$; 35 cycles of $30 \mathrm{~s}$ at $94^{\circ} \mathrm{C}, 1.5 \mathrm{~min}$ at $52{ }^{\circ} \mathrm{C}$, and $1 \mathrm{~min}$ at $72{ }^{\circ} \mathrm{C} ; 5 \mathrm{~min}$ at $72{ }^{\circ} \mathrm{C}$. PCR products were visualized in a $1 \%$ agarose gel and purified using ExoSap at $37^{\circ} \mathrm{C}$ for $15 \mathrm{~min}$, followed by $15 \mathrm{~min}$ at $80^{\circ} \mathrm{C}$. Bidirectional sequencing provided by service supplier (STAB Vida Ltd., Lisboa, Portugal). Obtained sequences were submitted to GenBank with the following accession numbers Ceratothoa aff. oestroides OK642789 and Cymodoce sp. OK646549.

\subsection{Data Analysis}

COI-5P sequences were edited and manually aligned using MEGA software (Kumar et al., 2018) and verified for the presence of stop codons, insertions or deletions. Homology searches were performed in BOLD database [35] and with BLASTn [36] in GenBank database [37]. Maximum Likelihood (ML) trees for the genus Cymodoce (dataset of 36 sequences (Table S1)) and Ceratothoa (dataset of 73 sequences (Table S1)) were constructed with MEGA software using HKY model with Gamma distribution with invariant sites $(\mathrm{HKY}+\mathrm{G}+\mathrm{I})$, chosen as the best-fit model of nucleotide substitutions by MEGA software. Bootstraps were performed using 10,000 replicates. Mined Ceratothoa oestroides sequences GQ240268 to GQ240280, from GenBank, were trimmed on the final 18 base pairs due to the presence of a stop codon followed by a shift on the reading frame. On the mined sequences from Ceratothoa italica JN604342 to JN604371 the same pattern was observed, and the final 118 base pairs were also trimmed. As no trace files are available for these public sequences, it is not possible to confirm these as pseudogenes or just errors on sequence editing or during submission to GenBank. Intra and interspecific divergence were calculated with the Kimura-2-Parameter (K2P) model as the standard genetic distance used in DNA barcode studies.

\section{Results}

A total of 98 specimens were examined belonging to 23 species, six from the order Decapoda, four from Cumacea, three from Mysida, two from Leptostraca, one from Tanaidacea and one from Balanomorpha. An update to the list of isopods from the Minho River [16] was also performed with the addition of six new records.

Subphyllum Crustacea Brünnich, 1772

Superclass Multicrustacea Regier, Shultz, Zwick, Hussey, Ball, Wetzer, Martin \& Cunningham, 2010

Class Malacostraca Latreille, 1802

Subclass Eumalacostraca Grobben, 1892

Superorder Eucarida Calman, 1904

Order Decapoda Latreille, 1802

Suborder Pleocyemata Burkenroad, 1963

Infraorder Astacidea Latreille, 1802

Superfamily Astacoidea Latreille, 1802

Family Cambaridae Hobbs, 1942

Genus Procambarus Ortmann, 1905 
Procambarus clarkii (Girard, 1852, [38]) (Figure 2A,B).

Synonym: Cambarus clarkii Girard, 1852: 91 [38]; Hagen, 1870: 39-41, Pl. I-IV [39]; Faxon, 1885: 26-27 [40].

Type material.

Holotype: Two male specimens collected in Monterrey, Mexico, deposited at Facultad de Ciencias Biológicas, Universidad Autónoma de Nuevo León, catalogue number UANLFCB-C3103984 [33].

Type locality: Texas, USA [38].

Material examined: Six megalopes size range 6 to $6.6 \mathrm{~mm}$, collected at the international Minho River, Vila Nova de Cerveira, $41^{\circ} 57^{\prime} 1.69^{\prime \prime} \mathrm{N} / 8^{\circ} 44^{\prime} 42.74^{\prime \prime} \mathrm{W}$, 21 December 2020 on fyke nets; deposited as NatMIP-CMDe-0039; 1 adult size $150 \mathrm{~mm}$, collected at the international Minho River, Vila Nova de Cerveira, $41^{\circ} 57^{\prime} 1.69^{\prime \prime}$ N $/ 8^{\circ} 44^{\prime} 42.74^{\prime \prime}$ W, 6 April 2021 on fyke nets; deposited as NatMIP-CMDe-0032.

Diagnosis: Antenna almost as long as body; rostrum triangular gradually tapering from the base, with two connected tubercles on the basis; anterior pair of pleopods with two rounded tubercles $[38,39]$.

Global distribution: Native to North America and Mexico [41]; introduced on Europe in Portugal, Spain, France, Austria, Cyprus, England, Germany, Italy, Netherlands and Switzerland [42]; on the Middle East in Israel [43]; on Africa in Kenya [44] and Uganda [45]; on South America in Brazil [46]; on Asia in China [47] and Japan [48].

Distribution in Portugal: Species recorded in the rivers Minho [49], Maçãs [50], Douro, Mondego, Sado, Caia [51], Tejo [52], Degebe [53], Alamos [54] and on São Miguel, Azores archipelago [42].

Ecological notes: Freshwater species, burrower, feeds on macrophytes [55].

Infraorder Brachyura Latreille, 1802

Superfamily Portunoidea Rafinesque, 1815

Family Carcinidae MacLeay, 1838

Genus Carcinus Leach, 1814

Carcinus maenas (Linnaeus, 1758) (Figure 2C-E).

Carcinus maenas Zariquiey-Alvarez, 1968: 354, Fig. 115A-C [25]; Hayward \& Ryland, 2017: 434, Fig. 8.64 [22].

Synonyms: Cancer maenas Linnaeus, 1758: 627 [56].

Cancer granarius Herbst, 1790: 107-108, Tab. II, Fig. 28a.A. [57].

Cancer viridis Herbst, 1790: 148, Tab. VII, Fig. 47. [57].

Cancer pygmeus Fabricius, 1787: 320 [58].

Cancer rhomboidalis Montagu, 1804: 65, Tab. VI, Fig. 1 [59].

Cancer granulatus Say, 1817: 61 [60].

Megalopa montagui Leach, 1815: Tab. XVI, Figs. 1-6 [61].

Portunus carcinoides Kinahan, 1857: 13 [62].

Type material: Unknown.

Type locality: Unknown.

Material examined: Six megalopes (size $3.2 \mathrm{~mm}$ ), collected at the international Minho River, Caminha $41^{\circ} 52^{\prime} 59.00^{\prime \prime}$ N $/ 8^{\circ} 50^{\prime} 14.00^{\prime \prime}$ W, 6 April 2020 on glass eel fishing bycatch; deposited as NatMIP-CMDe-0036; 4 juveniles, carapace length $12 \mathrm{~mm}$, same data as preceding, deposited as NatMIP-CMDe-0037; 1 adult, carapace length $58 \mathrm{~mm}$ collected at the international Minho River, Caminha $41^{\circ} 52^{\prime} 76.00^{\prime \prime}$ N $/ 8^{\circ} 50^{\prime} 41.70^{\prime \prime}$ W, 14 March 2021 on glass eel fishing bycatch deposited as NatMIP-CMDe-0038.

Diagnosis: Carapace frontal margin with three rounded lobes dactyl of pereopod 5 slightly broader than pereopods $2-4$ [22,25].

Global distribution: Native to Northeast Atlantic from Norway to North Africa [22]; introduced in Australia [63], Canada, USA, Japan, South Africa [64] and Argentina [65].

Distribution in Portugal: Common species along the Portuguese coast and estuaries $[13,66-68]$. 
Ecological notes: Intertidal species on estuaries, salt marshes and shallow coastal areas [22].

Infraorder Caridea Dana, 1852

Superfamily Atyoidea De Haan, 1849

Family Atyidae De Haan, 1849

Genus Atyaephyra de Brito Capello, 1866
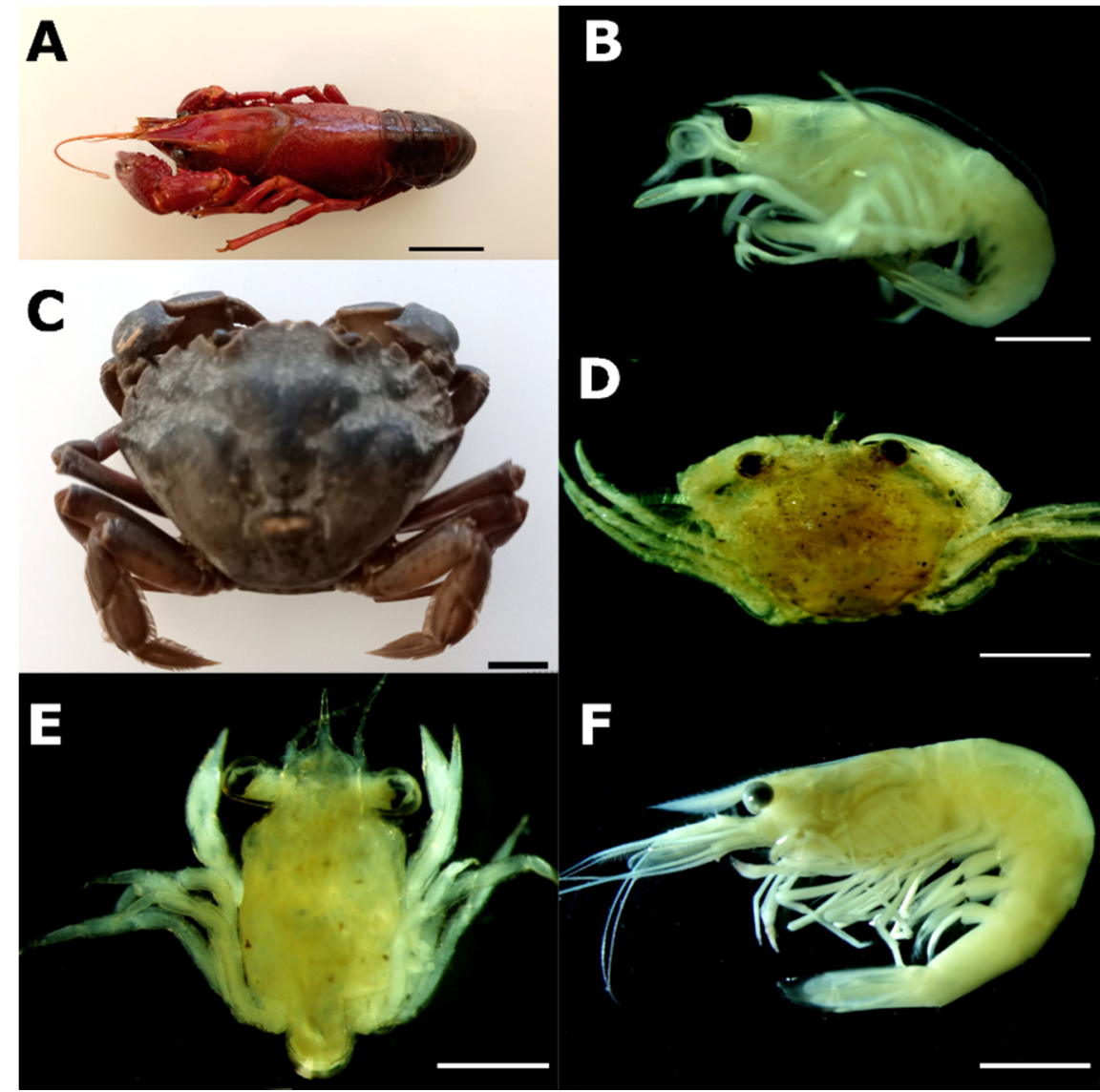

Figure 2. Procambarus clarkii (Girard, 1852): adult dorsal view (A), megalope side view (B); Carcinus maenas (Linnaeus, 1758): adult dorsal view (C), juvenile dorsal view (D), megalope dorsal view (E); and Atyaephyra desmarestii (Millet, 1831): adult side view (F). Scale bars: $(\mathbf{A})=2 \mathrm{~cm} ;(\mathbf{B}, \mathbf{E})=1 \mathrm{~mm}$; $(\mathbf{C})=1 \mathrm{~cm} ;(\mathbf{D}, \mathbf{F})=5 \mathrm{~mm}$.

Atyaephyra desmarestii (Millet, 1831) (Figure 2F).

Atyaephyra desmaresti Zariquiey-Alvarez, 1968: 79-81, Figs. 13C,D and 32A-D [25]; Anastasiadou et al., 2004: 6-13 [69]; Anastasiadou et al., 2006: 1196-1207, Figs. 1-5 [26]; Christodoulou et al., 2012: 66-71 [70].

Synonyms: Symethus fluviatilis Rafinesque, 1814: 23-24 [71].

Hippolyte desmarestii Millet, 1831: 56-57, Pl. 1, Fig. 1A,B [72].

Caridina desmarestii Joly, 1843: 46-82, Pl. 3 Figs. 1-78 [73].

Atyaephyra rosiana De Brito Capelo, 1866: 6-7, Pl. 1, Fig. 1 [74].

Atyaephyra desmaresti var. occidentalis Bouvier, 1913: 65-74, Figs. 1-3 [75].

Type material.

Neotype: One ovigerous female collected in Maine-et-Loire, France, deposited at Muséum National d'Histoire Naturelle, Paris, France (MNHN), catalogue number MNHNIU-2009-2270 [69].

Type locality: France [71]. 
Material examined: Six specimens, size range 17 to $27 \mathrm{~mm}$ collected at the international Minho River, Vila Nova de Cerveira, $41^{\circ} 57^{\prime} 1.69^{\prime \prime} \mathrm{N} / 8^{\circ} 44^{\prime} 42.74^{\prime \prime} \mathrm{W}$ on fyke nets, deposited as NatMIP-CMDe-0034.

Diagnosis: Rostrum long and slender, slightly curved upwards with 23-28 teeth (2-4 behind eye orbit) on upper margin and 5-10 in the lower margin [26,69].

Global distribution: West Europe and North Africa [69].

Distribution in Portugal: Present on the rivers Minho [13,14], Lima, Cávado, Ave, Douro, Mondego, Tejo and Guadiana [76].

Ecological notes: Omnivorous decapod present in freshwater $[69,75]$.

Superfamily Crangonoidea Haworth, 1825

Family Crangonidae Haworth, 1825

Genus Crangon Fabricius, 1798

Crangon (Linnaeus, 1758) (Figure 3A).

Crangon Zariquiey-Alvarez, 1968: 184-187, Figs. 77, and 78A,B [25]; Smaldon, 1993: 114, Fig. 42 [2]; Hayward \& Ryland, 2017: 408, Fig. 8.51 [22].

Synonyms: Cancer crangon Linnaeus, 1758: 632 [56].

Crangon vulgaris Fabricius, 1798: 410 [77].

Crangon rubro punctatus Risso, 1816: 85-86 [78].

Crangon maculosus Rathke, 1837: 366-368 [79]; Czerniavsky, 1884: 71 [80].

Cancer schillinus Nardo, 1847.

Steiracrangon orientalis Czerniavsky, 1884: 73-75 [80].

Type material: Unknown.

Type locality: Unknown.

Material examined: Five specimens, size range $20 \mathrm{~mm}$ to $50 \mathrm{~mm}$, collected at the international Minho River, Caminha $41^{\circ} 52^{\prime} 59.00^{\prime \prime}$ N/8 $50^{\prime} 14.00^{\prime \prime}$ W, 6 April 2020 on glass eel fishing bycatch; deposited as NatMIP-CMDe-0035.

Diagnosis: Rostrum unarmed; carapace with a spine on the anterior median line; pereopod 2 with 0.75 times the length of pereopod 1 propodus; pereopod 2 dactylus with half or more the length of propodus; telson with two pairs of lateral spines [2].

Global distribution: Northeast Atlantic from the Baltic Sea to Northwest Africa and Mediterranean Sea [22].

Distribution in Portugal: Species recorded on the estuaries of the rivers Minho [13,14,81], Lima [66], Cávado [67], Douro, Aveiro, Mira [82], Mondego [83] and Tejo [84], and at Fuzeta beach [85].

Ecological notes: Common on sandy bays and beaches and lower estuarine areas; species with commercial value [2].

Superfamily Palaemonoidea Rafinesque, 1815

Family Palaemonidae Rafinesque, 1815

Genus Palaemon Weber, 1795

Palaemon longirostris Milne Edwards, 1837 (Figure 3B,C).

Palaemon longirostris Milne Edwards, 1837: 394-395 [86]; Zariquiey-Alvarez, 1968: 168, Fig. 69B [25]; Smaldon, 1993: 36, Fig. 10 [2]; Hayward \& Ryland, 2017: 394, Fig. 8.44 [22].

Synonyms: Astacus albescens Pennant, 1812: 25-26 [87].

Palaemon edwardsii Heller, 1863: 265 [88].

Palaemon longipes Fischer, 1872: 421 [89].

Leander longirostris De Man, 1915: 149, Pl. 12, Fig. 3.

Palaemon garciacidi Zariquiey-Alvarez, 1968: 167, Figs. 69C, and 70 [25]. 


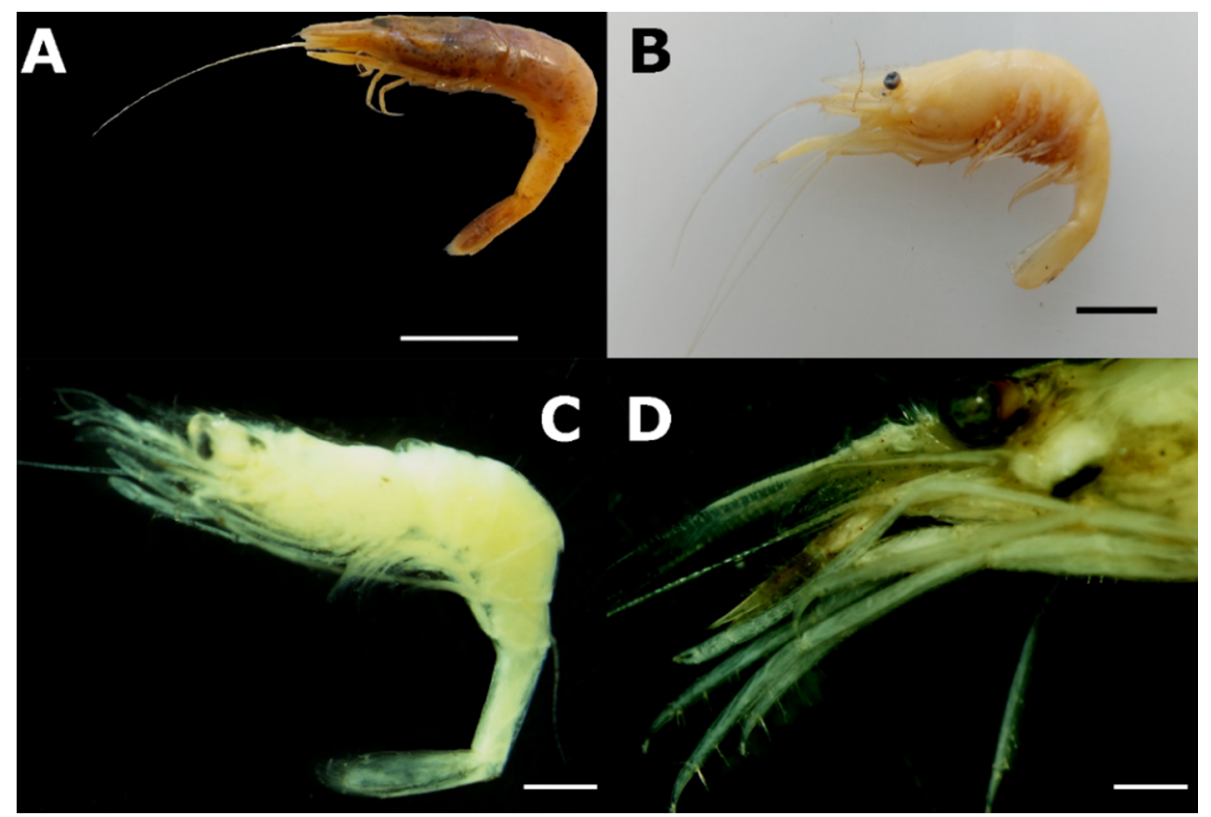

Figure 3. Crangon crangon (Linnaeus, 1758): adult side view (A); Palaemon longirostris Milne Edwards, 1837: adult side view (B), megalope side view (C); and Processa modica Williamson, 1979: adult pereopod 2 detail $(\mathbf{D})$. Scale bars: $(\mathbf{A}, \mathbf{B})=1 \mathrm{~cm} ;(\mathbf{C}, \mathbf{D})=1 \mathrm{~mm}$.

\section{Type material.}

Syntypes: Six specimens collected on Hollands Diep, Netherlands, deposited at Naturalis Biodiversity Center, catalogue number RMNH.CRUS.D.29725 [33].

Type locality: Unknown.

Material examined: Six megalopes, size range 9.9 to $12.2 \mathrm{~mm}$, collected at the international Minho River, Caminha, $41^{\circ} 52^{\prime} 59.00^{\prime \prime}$ N $/ 8^{\circ} 50^{\prime} 14.00^{\prime \prime}$ W, 6 April 2020; deposited as NatMIP-CMDe-0040 on glass eel fishing bycatch; two adults, one ovigerous female, size range 50 to $60 \mathrm{~mm}$, collected at the International Minho River, Caminha, $41^{\circ} 52^{\prime} 59.00^{\prime \prime} \mathrm{N} /$ $8^{\circ} 50^{\prime} 14.00^{\prime \prime}$ W, 6 April 2020 on glass eel fishing bycatch; deposited as NatMIP-CMDe-0041.

Diagnosis: Rostrum slightly upcurved with seven to eight dorsal teeth and three to five ventral teeth, two of the dorsal teeth behind the orbit; lower half of the rostrum without red-pigmented spots; pereopod 2 carpus equal to or slightly longer than merus [2,22].

Global distribution: Northeast Atlantic from Germany to Morocco and Mediterranean Sea [22,25].

Distribution in Portugal: Species recorded on the estuaries of the rivers Mira [90], Sado [91] and Tejo [92].

Ecological notes: On estuarine and brackish waters [22].

Superfamily Processoidea Ortmann, 1896

Family Processidae Ortmann, 1896

Genus Processa Leach, 1815

Processa modica Williamson, 1979 (Figure 3D).

Processa modica Williamson \& Rochanaburanon, 1979: 12-25, Figs. 1, 2, 3F-I, 4, 6A,D,G, and 9D [93]; Smaldon, 1993: 96, Fig. 36 [2].

Type material.

Paratype: Ten specimens on larval stage, collected at Dogger Bank, UK on 8 September 1976, deposited at Natural History Museum of London (NHMUK) catalogue number 1977.150 [33].

Type locality: The Hague, Netherlands [93].

Material examined: One adult specimen, size $12 \mathrm{~mm}$ length, collected at the international Minho River, Caminha $41^{\circ} 52^{\prime} 44.80^{\prime \prime}$ N, $8^{\circ} 50^{\prime} 26.25^{\prime \prime}$ W, 14 Mar. 2021 on glass eel fishing bycatch; deposited as NatMIP-CMDe-0047. 
Diagnosis: Carapace with an antennal spine, second pair of pereopods equal in length, pereopod 2 merus with 11 segments (Figure 3D) [2,93].

Global distribution: Northeast Atlantic from the Netherlands to the Iberian Peninsula and Mediterranean [2].

Distribution in Portugal: Species recorded along the Portuguese west coast [94].

Ecological notes: Marine; depth range from 0.5 to $100 \mathrm{~m}$; protandrous hermaphrodite [2].

Superorder Peracarida Calman, 1904

Order Cumacea Krøyer, 1846

Family Bodotriidae Scott, 1901

Genus Iphinoe Bate, 1856

Iphinoe tenella Sars, 1878 (Figure $4 \mathrm{~A}, \mathrm{~B})$.

Iphinoe tenella Sars, 1878: 505-506, Pls. 15-16 [95]; Stebbing, 1913: 47 [96]; Ledoyer, 1965: 259-260, Pl. II Figs. 2A,B, and 3A,B, Pl. IV Fig. 1A,B, Pl. VI, Figs. 2-3, Pl. VII, Fig. 1, Pl. X, Figs. 2-3, Pl. XII, Fig. 1, Pl. XIV, Figs. 2,3, Pl. XVI, Fig. 1 [27]; Jones, 1976: 22, Fig. 6K-N [23]; Corbera \& Garcia-Rubies, 1998: Fig. 4A [97]; Mazziotti \& Lezzi, 2020: 347-348 [28].

Type material: Unknown.

Type locality: Siracusa, Italy [95].

Material examined: Six adult specimens, four female (size $11 \mathrm{~mm}$ ) and two males, size range 9.4 to $10.1 \mathrm{~mm}$, collected at the international Minho River, Caminha $41^{\circ} 52^{\prime} 59.00^{\prime \prime} \mathrm{N} /$ $8^{\circ} 50^{\prime} 14.00^{\prime \prime}$ W, 6 April 2020 on glass eel fishing bycatch; deposited as NatMIP-CMCu-0049.

Diagnosis: Male and female with teeth on dorsal midline of carapace; antennula with a single aesthtasc; antenna as long as the body size on male; telson with six perianal setae [27].

Global distribution: Atlantic Ocean from British isles to the Iberian Peninsula and Mediterranean [22,23].

Distribution in Portugal: Species recorded on Troia [98], Alvor estuary, Lagoa de Albufeira [99] and Ria Formosa [100,101].

Ecological notes: From intertidal to shallow sublittoral, on muddy sand [22].

Iphinoe trispinosa (Goodsir, 1843) (Figure 4C,D).

Iphinoe trispinosa Ledoyer, 1965: 259, Pl. III Fig. 1A,B, Pl. VII Fig. 1, Pl. XI Fig. 1, Pl. XV Fig. 1 [27]; Jones, 1976: 22, Fig. 6A-E [23]; Hayward \& Ryland, 2017: 315, Fig. 8.13 [22]; Mazziotti \& Lezzi, 2020: 348-351 [28].

Synonyms: Cuma trispinosa Goodsir, 1843: 126-127, Pl. II Fig. 19, Pl. III Fig. 1, Pl. IV Fig. 16 [102].

Halia trispinosa Bate, 1856: 459-460, Pl. XIV, Fig. V [103].

Venilia gracilis Bate 1856: 460, Pl. XV, Fig. VII [103].

Type material.

Holotype: Collected at Firth of Forth, Scotland; deposited at Natural History Museum of London (NHMUK), catalogue number 1966.12.16.1 [33].

Type locality: Firth of Forth, Scotland [33].

Material examined: Five adult specimens, four female (size $9 \mathrm{~mm}$ ) and one male (size $11.2 \mathrm{~mm}$ ), collected at the international Minho River, Caminha $41^{\circ} 52^{\prime} 59.00^{\prime \prime} \mathrm{N} / 8^{\circ} 50^{\prime} 14.00^{\prime \prime} \mathrm{W}$, 6 April 2020 on glass eel fishing bycatch; deposited as NatMIP-CMCu-0048 and NatMIPCMCu-0050.

Diagnosis: Female with two to six teeth on dorsal midline of carapace, smooth in male; antennula with a single aesthtasc; antenna as long as the body size on male; telson with two perianal setae [22,27].

Global distribution: Northeast Atlantic Ocean from Norway to the Iberian Peninsula and Mediterranean Sea [22].

Distribution in Portugal: Species recorded along the west coast $[94,99,104]$.

Ecological notes: From coastal intertidal up to depths of $150 \mathrm{~m}$, on fine sand [22]. 
Family Diastylidae Bate, 1856

Genus Diastylis Say, 1818

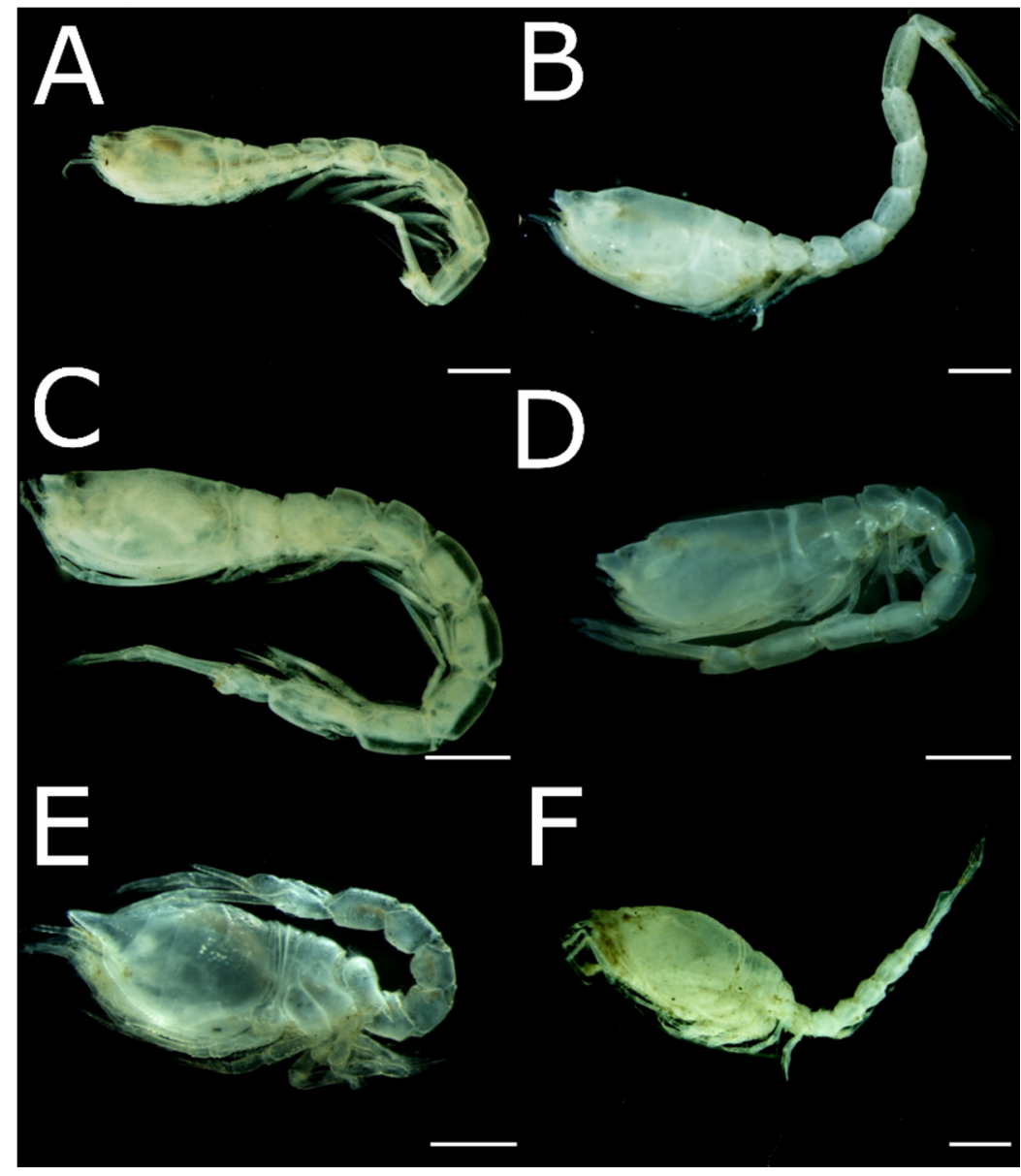

Figure 4. Iphinoe tenella Sars, 1878: male side view (A), female side view (B); Iphinoe trispinosa (Goodsir, 1843): male side view (C), female side view (D); Diastylis bradyi Norman, 1879 (E); and Diastylis cornuta (Boeck, 1864) (F). Scale bars: $(\mathbf{A}-\mathbf{F})=1 \mathrm{~mm}$.

Diastylis bradyi Norman, 1879 (Figure 4E).

Diastylis bradyi Norman, 1879: 59-60 [105]; Jones, 1976: 52, Fig. 17F-J [23].

Type material: Unknown.

Type locality: Lough Swilty, Donegal, Ireland [105].

Material examined: Six specimens, two male adults, size range 12.8 to $13.5 \mathrm{~mm}$ and four female adults, size range 9 to $10.1 \mathrm{~mm}$, collected at the international Minho River, Caminha $41^{\circ} 52^{\prime} 59.00^{\prime \prime} \mathrm{N} / 8^{\circ} 50^{\prime} 14.00^{\prime \prime} \mathrm{W}, 6$ April 2020 on glass eel fishing bycatch; deposited as NatMIP-CMCu-0051.

Diagnosis: Female carapace with denticles; postero-lateral corners of pereonite 5 with acute prolongations; propodus less than twice as long as dactylus [23].

Global distribution: Northeast Atlantic from Skagerak to Bay of Biscay [23].

Distribution in Portugal: Species recorded along the Portuguese southwest coast [99].

Ecological notes: On coarse sediments; depth range 0-30 m depth [23].

Diastylis cornuta (Boeck, 1863) (Figure 4F).

Diastylis cornuta Norman, 1879: 55 [105]; Sars, 1900: 45-47, Pls. XXXVI-XXXVII [106]; Jones, 1976:56, Fig. 19A-C [23].

Synonyms: Cuma cornuta Boeck, 1863: 190 [107].

Diastylis bispinosa Sars, 1864: 164-166 [108].

Diastylis cornutus Stebbing, 1913: 96-97, Figs. 55-57 [96].

Type material: Unknown. 
Type locality: Unknown.

Material examined: Two male adult specimens, size range 8 to $13.2 \mathrm{~mm}$, collected at the international Minho River, Caminha $41^{\circ} 52^{\prime} 59.00^{\prime \prime}$ N/8 $50^{\prime} 14.00^{\prime \prime}$ W, 6 April 2020 on glass eel fishing bycatch; deposited as NatMIP-CMCu-0052.

Diagnosis: Female carapace with numerous spines with two pairs specially large, reduced to tubercles on males; pleonites with various small spines; telson with 8 to 10 pairs of spines on each side [106].

Global distribution: Northeast Atlantic from Norway to the Iberian Peninsula and Mediterranean [33].

Distribution in Portugal: Species recorded at Portinho da Arrábida [99].

Ecological notes: Marine on soft bottom [104].

Order Mysida Boas, 1883

Family Mysidae Haworth, 1825

Genus Gastrosaccus Norman, 1868

Gastrosaccus spinifer (Goës, 1864) (Figure 5A).

Gastrosaccus spinifer Norman, 1892: 154 [109]; Zimmer, 1909: 61, Figs. 104-107 [31]; Makings, 1977: 588, Fig. 2K [30]; Hayward \& Ryland, 2017: 323, Fig. 8.18B [22].

Synonyms: Mysis spinifera Goës, 1864: 174-175 [110].

Acanthocaris livingstoneana Sim, 1872: 185-186, Pl. IV Fig. B [111].

Gastrosaccus spiniferus Stebbing, 1880: 114-118, Pl. III [112].

Type material: Unknown.

Type locality: Gullmaren, Sweden [110].

Material examined: Six adult specimens, size range 13 to $17 \mathrm{~mm}$, collected at the international Minho River, Caminha $41^{\circ} 52^{\prime} 59.00^{\prime \prime} \mathrm{N} / 8^{\circ} 50^{\prime} 14.00^{\prime \prime} \mathrm{W}, 6$ April 2020 on glass eel fishing bycatch; deposited as NatMIP-CMMy-0043.

Diagnosis: Dorsal fifth segment with a dorsal ridge forming a spine visible on lateral view $[22,30,31]$.

Global distribution: Northeast Atlantic from Baltic Sea to Northwest Africa and Mediterranean Sea [22].

Distribution in Portugal: Common species along the Portuguese coast and estuaries $[12,94,113]$.

Ecological notes: Benthic on coastal shallow to deep waters [30].

Genus Neomysis Czerniavsky, 1882

Neomysis integer (Leach, 1814) (Figure 5B).

Neomysis integer Tattersall \& Tattersall, 1951: 399-409, Figs. 109-110 [114]; Makings, 1977: 592, Fig. 2V [30]; Hayward \& Ryland, 2017: 330, Fig. 8.19F [22].

Synonyms: Praunus integer Leach, 1814: 401-402 [115].

Mysis integer Leach, 1815: Figs. 7-8 [61].

Mysis scoticus Thompson, 1829: 30-31 [116].

Mysis vulgaris Thompson, 1829: 30, Pl. 1 [116].

Neomysis vulgaris Czerniavsky, 1882: Pl. XVIII Figs. 18-22, Pl.XXX Figs. 12-14 [117]; Zimmer, 1909: 166-168, Figs. 368-373 [31].

Type material: Unknown.

Type locality: Lochranza, Scotland [115].

Material examined: Three adult specimens, size range 18.4 to $19.7 \mathrm{~mm}$, collected at the international Minho River, Caminha $41^{\circ} 52^{\prime} 59.00^{\prime \prime}$ N $/ 8^{\circ} 50^{\prime} 14.00^{\prime \prime}$ W, 6 April 2020 on glass eel fishing bycatch; deposited as NatMIP-CMMy-0042.

Diagnosis: Antennal scale tapering to a point, with setae all round; telson lateral sides converging gradually to a narrow almost pointed apex; uropods less than twice as long; endouropod with a comb of spines on the inner ventral side [22,30].

Global distribution: Northeast Atlantic [22].

Distribution in Portugal: Species recorded at Ria de Aveiro [118], Cávado [67] and Tejo [119] rivers. 
Ecological notes: Common in estuaries, brackish pools, on hypersaline pools up to freshwater and all intermediate salinity concentrations; occasionally on the open sea [22,30].

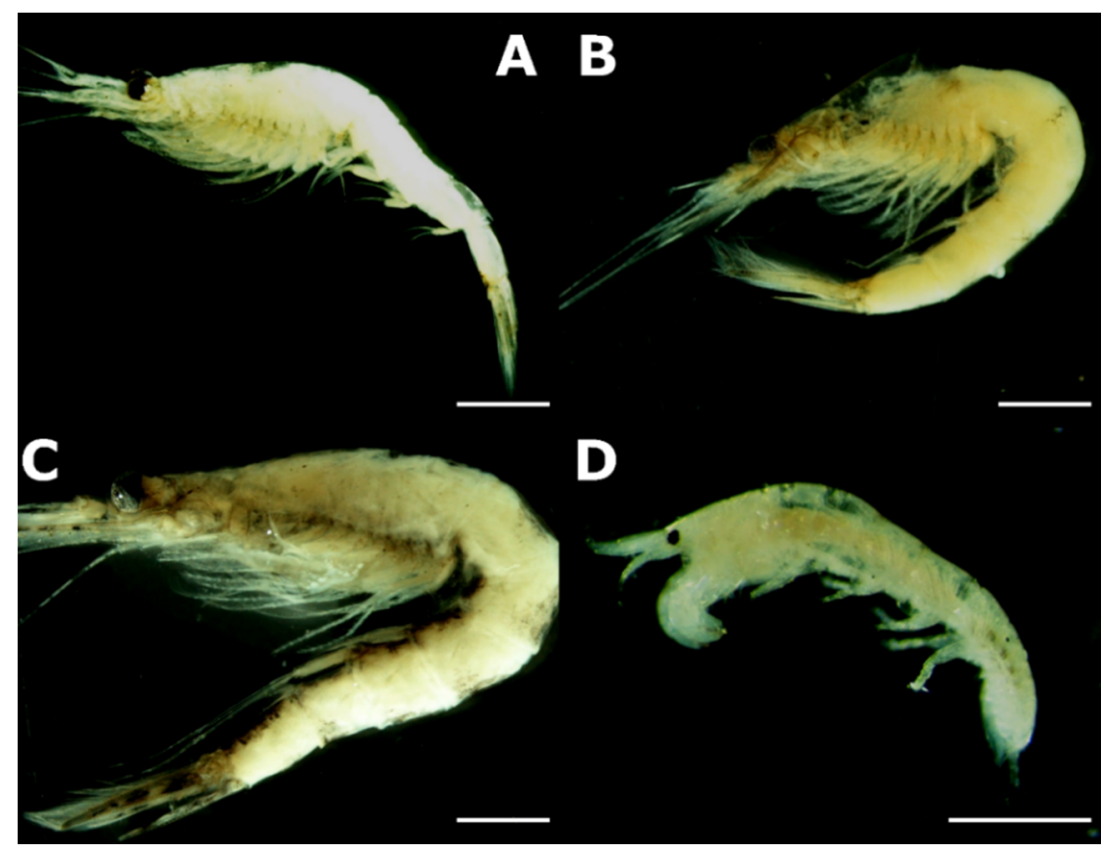

Figure 5. Gastrosaccus spinifer (Goës, 1864): side view (A); Neomysis integer (Leach, 1814): side view (B); Praunus neglectus (Sars, 1869): side view (C); and Heterotanais oerstedii (Krøyer, 1842): female side view (D). Scale bars: $(A-C)=2 \mathrm{~mm} ;(\mathbf{D})=0.5 \mathrm{~mm}$.

Genus Praunus Leach, 1814

Praunus neglectus (Sars, 1869) (Figure 5C).

Praunus neglectus Makings, 1977: 591, Fig. 2A-B [30]; Hayward \& Ryland, 2017: 326, Fig. 8.17A [22].

Synonyms: Mysis neglecta Sars, 1869: 341 [120].

Macromysis neglecta Norman, 1892: 252-253 [109].

Synmysis neglecta Czerniavsky, 1882: 26 [117].

Macromysis nigra Keeble \& Gamble, 1904 [121].

Type material: Unknown.

Type locality: Christiania, Denmark [120].

Material examined: Four adult specimens, size range 20 to $29 \mathrm{~mm}$, collected at the International Minho River, Caminha $41^{\circ} 52^{\prime} 59.00^{\prime \prime} \mathrm{N} / 8^{\circ} 50^{\prime} 14.00^{\prime \prime} \mathrm{W}, 6$ April 2020 on glass eel fishing bycatch; deposited as NatMIP-CMMy-0044.

Diagnosis: Antennal scale five to six times as long as broad; anterior lobe slightly longer than subterminal spine; telson with 18-24 lateral spines [22,30].

Global distribution: Northeast Atlantic [22].

Distribution in Portugal: Species recorded in Ria de Aveiro [122].

Ecological notes: Associated with weeds on shallow waters [30].

Order Tanaidacea Dana, 1849

Suborder Tanaidomorpha Sieg, 1980

Family Leptocheliidae Lang, 1973

Genus Heterotanais Sars, 1882

Heterotanais oerstedii (Krøyer, 1842) (Figure 5D).

Heterotanais oerstedii Norman, 1906: 169, Pl. VI Figs. 2-3 [123]; Holdich \& Jones, 1983: Figs. 4A,B, and 6A [124]; Barnes, 1994: 169, Fig. 75 [24]; Hayward \& Ryland, 2017: 321, Fig. 8.15 [22].

Synonyms: Tanais oerstedii Krøyer, 1842: 183-184 [125]. 
Tanais curculio Krøyer, 1842: 184-185 [125].

Tanais balticus Müller, 1852: 89-90 [126].

Tanais rhynchites Müller, 1852: 88-89 [126].

Heterotanais gurneyi Norman, 1906: 168-169, Pl. V, Pl. VI Fig. 1 [123].

Type material.

Syntypes: Collected in Norfolk, England in 1907; deposited at Natural History Museum of London (NHMUK), catalogue numbers 1956.10.10.104-108 and 1911.11.8.7243-7262 [33].

Type locality: Öresund, Baltic Sea [125].

Material examined: Three female specimens size $1.9 \mathrm{~mm}$, collected at the saltmarsh of the international Minho River, Caminha $41^{\circ} 52^{\prime} 30.42^{\prime \prime} \mathrm{N} / 8^{\circ} 49^{\prime} 52.97^{\prime \prime} \mathrm{W}$, March 2006 with Van Veen grab sampler; deposited as NatMIP-CMTa-0053.

Diagnosis: Male with anterior part of the carapace abruptly narrower than posterior part; eyes prominent on both sexes; antenna 1 with three articles on female and five on male; uropod exopodite with two articles and endopodite with four [22].

Global distribution: Northeast Atlantic from Norway to northern France [22].

Distribution in Portugal: Species recorded at the Minho River estuary [15], Ria de Aveiro [113] and Tejo estuary [99].

Ecological notes: From coastal to brackish habitats, constructing tubes on algae and hydroids, or on muddy substrates [22,24].

Subclass Phyllocarida Packard, 1879

Order Leptostraca Claus, 1880

Suborder Nebaliacea Calman, 1904

Family Nebaliidae Samouelle, 1819

Genus Nebalia Leach, 1814

Nebalia strausi Risso, 1826 (Figure 6A).

Nebalia strausi Risso, 1826: 84-85 [127]; Dahl, 1985: 155-157, Figs. 63-78 [128]; Moreira et al., 2004: 84-92, Figs. 1-6 [129]; Moreira et al., 2009a: 271, Fig. 1E [130]; Moreira et al., 2009b: 101-102, Pl. 1, Figs. 1-3 [29]; McCormack et al., 2016: Fig. 3D [131].

Type material.

Lectotype: One female from Bay of Naples, Italy deposited at Natural History Museum of London (NHMUK) [129].

Type locality: Unknown.

Material examined: Three adult specimens, size range 7.1 to $8.7 \mathrm{~mm}$, collected at the international Minho River, Caminha $41^{\circ} 52^{\prime} 59.00^{\prime \prime} \mathrm{N} / 8^{\circ} 50^{\prime} 14.00^{\prime \prime} \mathrm{W}, 6$ April 2020 on glass eel fishing bycatch; deposited as NatMIP-CMLe-0046.

Diagnosis: Pleonites 6-7 with distally acute denticles; antennular flagellum with more than 10 articles; antennular scale more than twice as long as wide [29].

Global distribution: Northeast Atlantic from northwest France and British isles to the Iberian Peninsula and Mediterranean Sea [29,131].

Distribution in Portugal: New record (this study).

Ecological notes: On coarse sediments and rhodoliths-environments, at depths of 4-23 m [2,131].

Genus Sarsinebalia Dahl, 1985 


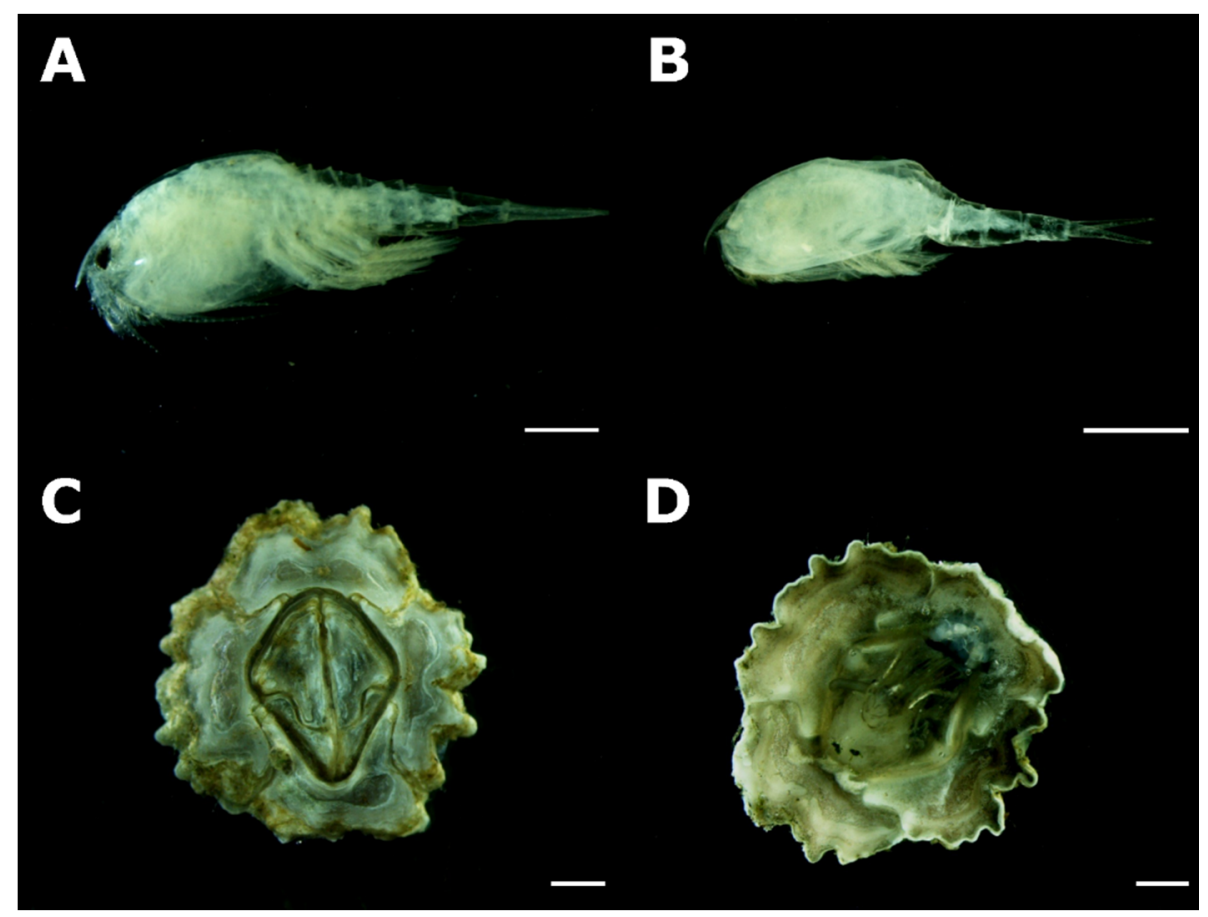

Figure 6. Nebalia strausi Risso, 1826: side view (A); Sarsinebalia cristoboi Moreira, Gestoso \& Troncoso, 2003: side view (B); Austrominius modestus (Darwin, 1854): dorsal view (C), and ventral view (D). Scale bars: $(\mathbf{A}, \mathbf{C}, \mathbf{D})=1 \mathrm{~mm} ;(\mathbf{B})=2 \mathrm{~mm}$.

Sarsinebalia cristoboi Moreira, Gestoso \& Troncoso, 2003 (Figure 6B).

Sarsinebalia cristoboi Moreira et al., 2003: 191-200, Figs. 1-7, 15A [132]; Moreira et al., 2009a: 282, Fig. 1C [29].

Type material.

Holotype: 1 female collected at Ria de Vigo, Spain $42^{\circ} 13^{\prime} 54^{\prime \prime} \mathrm{N} 8^{\circ} 46^{\prime} 30^{\prime \prime} \mathrm{W}$ on October 2000, 14 m depth; deposited at Museo Nacional de Ciencias Naturales, Madrid, catalogue number MNCN 20.04/5326a [132].

Paratypes: Three females collected at Ria de Vigo, Spain $42^{\circ} 13^{\prime} 54^{\prime \prime} \mathrm{N} 8^{\circ} 46^{\prime} 30^{\prime \prime} \mathrm{W}$ on October 2000, $14 \mathrm{~m}$ depth; deposited at Museo Nacional de Ciencias Naturales, Madrid, catalogue number MNCN 20.04/5326c [132].

Type locality: Ria de Vigo, Spain [132].

Material examined: One adult specimen, size $8.5 \mathrm{~mm}$, collected at the International Minho River, Caminha $41^{\circ} 52^{\prime} 59.00^{\prime \prime}$ N/8 $50^{\prime} 14.00^{\prime \prime}$ W, 6 April 2020 on glass eel fishing bycatch; deposited as NatMIP-CMLe-0045.

Diagnosis: Antennal flagellum with thin long setae; supraorbital scale extending beyond the distal end of the eye; pigmented eye; pleonite 4 with acute denticles [29].

Global distribution: Only known from the west Iberian coast [94,132].

Distribution in Portugal: Species recorded along the Portuguese west coast with a southern limit at Aljezur [94].

Ecological notes: Collected at depths between 9 and $33 \mathrm{~m}$, coarse sand bottoms with a high presence of red non-geniculate calcareous algae (Rhodoliths) [132].

Class Thecostraca Gruvel, 1905

Subclass Cirripedia Burmeister, 1834

Superorder Thoracicalcarea Gale, 2015

Order Balanomorpha Pilsbry, 1916

Superfamily Elminioidea Chan, Dreyer, Gale, Glenner, Ewers-Saucedo, Pérez-Losada, Kolbasov, Crandall \& Høeg, 2021

Family Elminiidae Foster, 1982

Genus Austrominius Buckeridge, 1983 
Austrominius modestus (Darwin, 1854) (Figure 6C,D).

Austrominius modestus Hayward \& Ryland, 2017: 302, Fig. 8.8 [22].

Synonyms: Elminius modestus Darwin, 1854: 350-351, Pl. 12 Figs. 1A-E [133]; Foster, 1978: 95, Pl. 12C Fig. 57 [134]; Barnes, 1994: 154, Fig. 67 [24].

Elminius sinuatus Hutton, 1879: 328 [135].

Type material.

Lectotype: Collected in New Zealand, deposited at NHMUK catalogue number 1981.274 [33].

Type locality: New Zealand [133].

Material examined: One specimen, diameter $6 \mathrm{~mm}$, collected at the international Minho River, Caminha $41^{\circ} 52^{\prime} 00^{\prime \prime} \mathrm{N} / 8^{\circ} 51^{\prime} 15.90^{\prime \prime} \mathrm{W}$, March 2021, on rock, deposited as NatMIP-CTBa-0055.

Diagnosis: Shell with four wall plates, low conical shape, orifice diamond-shaped [22,133].

Global distribution: Native to New Zealand and introduced to northwest Europe, present from Britain to south Portugal [22].

Distribution in Portugal: Species recorded at Setúbal, Faro [136] and Madeira island [137].

Ecological notes: Estuarine and costal, intertidal and shallow subtidal, on rocks, shells, algae, other crustaceans and artificial structures [22].

Update to Isopods from the Minho River [14]:

Order Isopoda Latreille, 1817

Suborder Asellota Latreille, 1802

Superfamily Janiroidea Sars, 1897

Family Janiridae Sars, 1897

Genus Jaera Leach, 1814

Jaera (Jaera) albifrons Leach, 1814 (Figure 7A,B).

Jaera (Jaera) albifrons Kussakin, 1988: 138-142, Figs. 115-117 [138]; Hayward \& Ryland, 2017: 347-348, Fig. 8.25 [22].

Synonyms: Jaera albifrons Leach, 1814: 434 [115]; Hansen, 1916:12-13 [139]; Naylor, 1972: 54, Figs. 15A,B, 16A, and 17A [6].

Oniscus marinus Fabricius, 1780: 252 [140].

Jaera nivalis Kröyer, 1838: 303-306, Table IV, Fig. 21 [141].

Jaera marina Sars, 1899: 104-105, Pl. XLIII [142]; Richardson, 1905: 450-451, Figs. 503-504 [143].

Jaera albifrons albifrons Forsman, 1949: 455-456, Figs. 3-4 [144].

Type material: Unknown.

Type locality: Greenland [140].

Material examined: One adult male, size $2.33 \mathrm{~mm}$ length, collected the international Minho River, Caminha $41^{\circ} 52^{\prime} 04.8^{\prime \prime}$ N 8 $51^{\prime} 18.8^{\prime \prime} \mathrm{W}$, June 2021 on beam trawl, deposited as NatMIP-CMIs-0054.

Diagnosis: Body oval in females, males smaller than females with eyes relatively large; lateral margins of both sexes fairly sparsely fringed with spines; pereopods 6 and 7 of male with the distal region of carpus extended as a lobe fringed with spines (Figure 7B) [6].

Global distribution: Northeast Atlantic from Russia to France and northwest Atlantic from Greenland to Maine (USA) [6].

Distribution in Portugal: Species recorded along the Portuguese west coast [113,145].

Ecological notes: Common on sheltered shores, occurring often in estuaries usually beneath stones [6].

Suborder Cymothoida Wägele, 1989

Superfamily Cymothooidea Leach, 1814

Family Cymothoidae Leach, 1818

Genus Ceratothoa Dana, 1852 


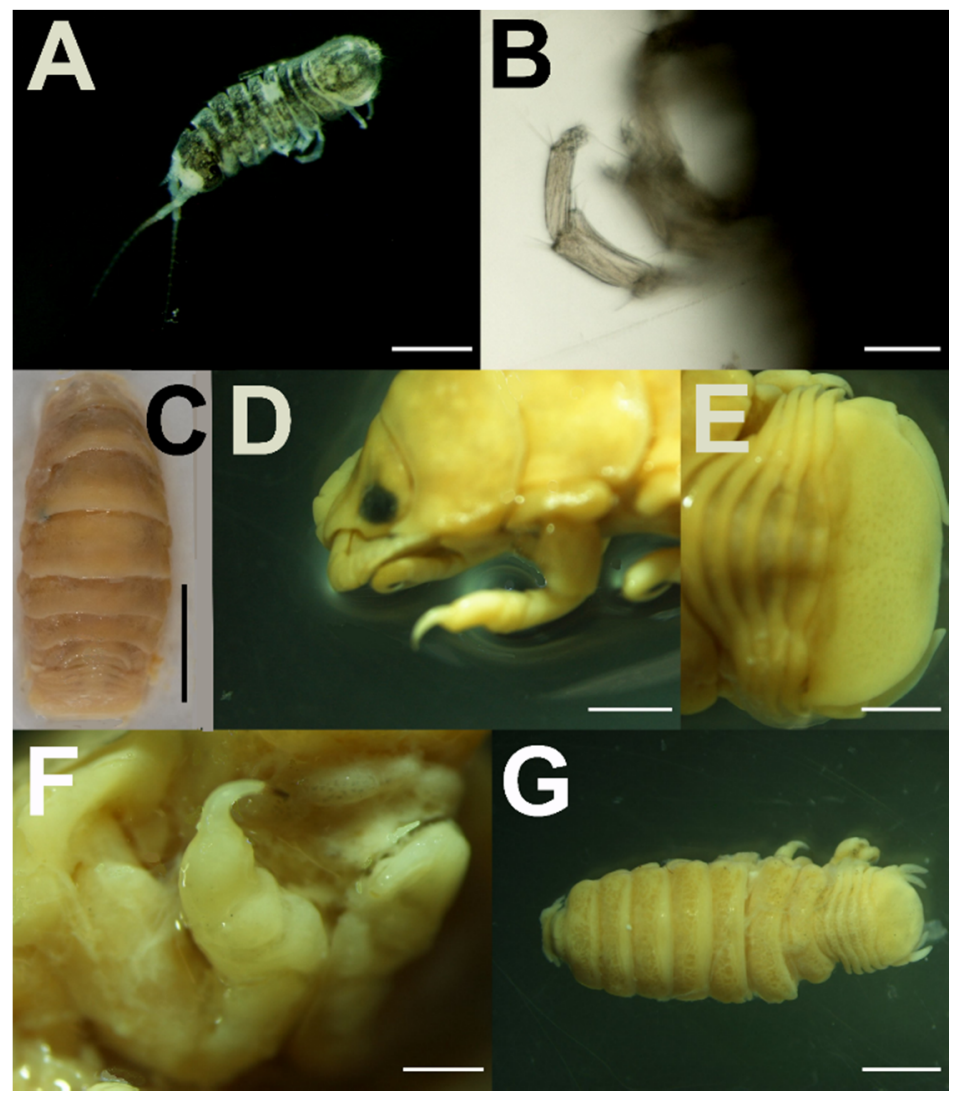

Figure 7. Jaera (Jaera) albifrons Leach, 1814: dorsal view (A), pereopod 6 (B); Ceratothoa aff. oestroides (Risso, 1826): dorsal view (C), cephalon lateral view (D), pleotelson $(\mathbf{E})$, pereopod $1(\mathbf{F})$, and juvenile (G). Scale bars: $(\mathbf{A})=0.5 \mathrm{~mm} ;(\mathbf{B})=0.05 \mathrm{~mm} ;(\mathbf{C})=1 \mathrm{~cm} ;(\mathbf{D})=1 \mathrm{~mm} ;(\mathbf{E}-\mathbf{G})=2 \mathrm{~mm}$.

Ceratothoa aff. oestroides (Risso, 1826) (Figure 7C-F).

Ceratothoa oestroides Meinert \& Schiødte, 1879: 350-356, Table XV (Cym. XXII), Figs. 5-11 [146]; Carus, 1885: 442 [147]; Barrois, 1888: 63 [148]; Sociedad Española de historia natural, 1890: 118 [149]; Gourret, 1891: 14-15, Pl. IV, Figs. 10-11 [150]; Koelbel, 1892: 107, 115 [151]; Graeffe, 1902: 26 [152]; Brian, 1902; 15 [153]; Nobre, 1903a: 61 [154]; Nobre, 1903b: 156 [155]; Trilles, 1991: 122-124 [156]; Horton, 2000: 1047-1048, Fig. 7A-B [9]; Hadfield et al., 2016: 65-69, Fig. 9 [157].

Synonyms: Canolira oestroides Risso, 1826: 123 [127].

Cymothoa oestroides Milne Edwards, 1840: 272 [158]; Lucas, 1849: 78, Pl. 8, Fig. 3 [159]. Ceratothoa sargorum Gourret, 1891: 16, Pl. I, Fig. 17, Pl. V, Figs. 1-4 [150].

Meinertia oestroides Nierstrasz, 1915: 89 [160]; Trilles, 1972: 1201-1208, Figs. 90-136, Pl. I, Figs. 6-9, Pl. III, Fig. 20 [161]; Trilles \& Raibaut, 1973: 274 [162].

Type material

Holotype: Specimen collected at Nice, France [156].

Syntypes: Two females collected in Nice, France deposited at Musée d'Histoire Naturelle, Paris, catalogue number MNHN-Is431 [9].

Lectotype: One female collected at the Mediterranean Sea, deposited at National Museum of Natural History, Paris, catalogue number MNHN-IU-2014-17478 [157].

Paralectotype: Female specimen deposited at National Museum of Natural History, Paris, catalogue number MNHN-IU-2007-4240 [157].

Type locality: Nice, France [156].

Material examined: One adult female with $30 \mathrm{~mm}$ deposited as NatMIP-CMIs-0059 with COI-5P sequence (pereopod 1 missing); one adult female and one juvenile with $30 \mathrm{~mm}$ and $9.8 \mathrm{~mm}$ deposited as NatMIP-CMIs-0060; one adult male with $26 \mathrm{~mm}$ deposited as NatMIP-CMIs-0061; one ovigerous female with $29 \mathrm{~mm}$ deposited as NatMIP-CMIs-0062. 
All specimens were collected on the mouth of Alosa alosa (Linnaeus, 1758) in Vila Nova de Cerveira, International Minho River $\left(41^{\circ} 55^{\prime} 57.67^{\prime \prime} \mathrm{N} / 8^{\circ} 45^{\prime} 33.85^{\prime \prime} \mathrm{W}\right)$ in May 2013.

Diagnosis: Rostrum acute; pereonite 1 anterolateral angle extending to the middle of the eye (Figure 7D); expansions on merus of pereopods 1-3 (Figure 7F); uropod slightly longer than pleotelson (Figure 7E) $[9,157]$.

Global distribution: Mediterranean Sea and the east Atlantic Ocean from north Africa to France $[9,157]$

Distribution in Portugal: Species recorded in Póvoa de Varzim, Matosinhos [154], Faro and on Azores archipelago [148].

Ecological notes: Parasitic on fishes: Boops boops (Linnaeus, 1758); Diplodus sargus (Linnaeus, 1758); Diplodus annularis (Linnaeus, 1758); Diplodus vulgaris (GeoffroySaint-Hilaire, 1817); Diplodus bellottii (Steindachner, 1882); Spicara maena (Linnaeus, 1758); Spicara smaris (Linnaeus, 1758); Mullus barbatus barbatus Linnaeus, 1758; Trachurus trachurus (Linnaeus, 1758); Trachurus mediterraneus (Steindachner, 1868); Phycis (Linnaeus, 1766); Pagellus acarne (Risso, 1827); Pagellus erythrinus (Linnaeus, 1758); Sardina pilchardus (Walbaum, 1792); Scorpaena notata Rafinesque, 1810; Scorpaena porcus Linnaeus, 1758; Scorpaena scrofa Linnaeus, 1758; Spondyliosoma cantharus (Linnaeus, 1758); Uranoscopus scaber Linnaeus, 1758; Abudefduf saxatilis (Linnaeus, 1758); Argyrosomus regius (Asso, 1801); Pagellus erythrinus (Linnaeus, 1758); Dicentrarchus labrax (Linnaeus, 1758); Sparus aurata Linnaeus, 1758; Lithognathus mormyrus (Linnaeus, 1758); Pomatomus saltatrix (Linnaeus, 1766); Rostroraja alba (Lacepède, 1803); Zeus faber Linnaeus, 1758 [163].

Family Gnathiidae Leach, 1814

Genus Gnathia Leach, 1814

Gnathia vorax (Lucas, 1849) (Figure 8A,B).

Gnathia vorax Naylor, 1957: 3, Fig. 5A,B [164]; Naylor, 1972: 19, Fig. 4G [6].

Synonyms: Anceus vorax Lucas, 1849: 85-86 [159].

Type material: Unknown.

Type locality: Algerie [159].

Material examined: One juvenile (praniza) size $4.73 \mathrm{~mm}$ length, collected the inter-

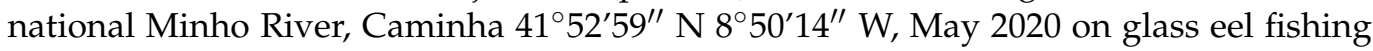
bycatch, deposited as NatMIP-CMIs-0058.

Diagnosis: Male cephalon with central concavity and an acute median projection [165].

Global distribution: North Atlantic from Greenland to the British Isles extending southwards to the Mediterranean [6].

Distribution in Portugal: Species recorded on the Mondego estuary [99] and in Sines [166].

Ecological notes: Pranizas ectoparasitic on fish [167].

Suborder Valvifera Sars, 1883

Family Idoteidae Samouelle, 1819

Genus Idotea Fabricius, 1798 


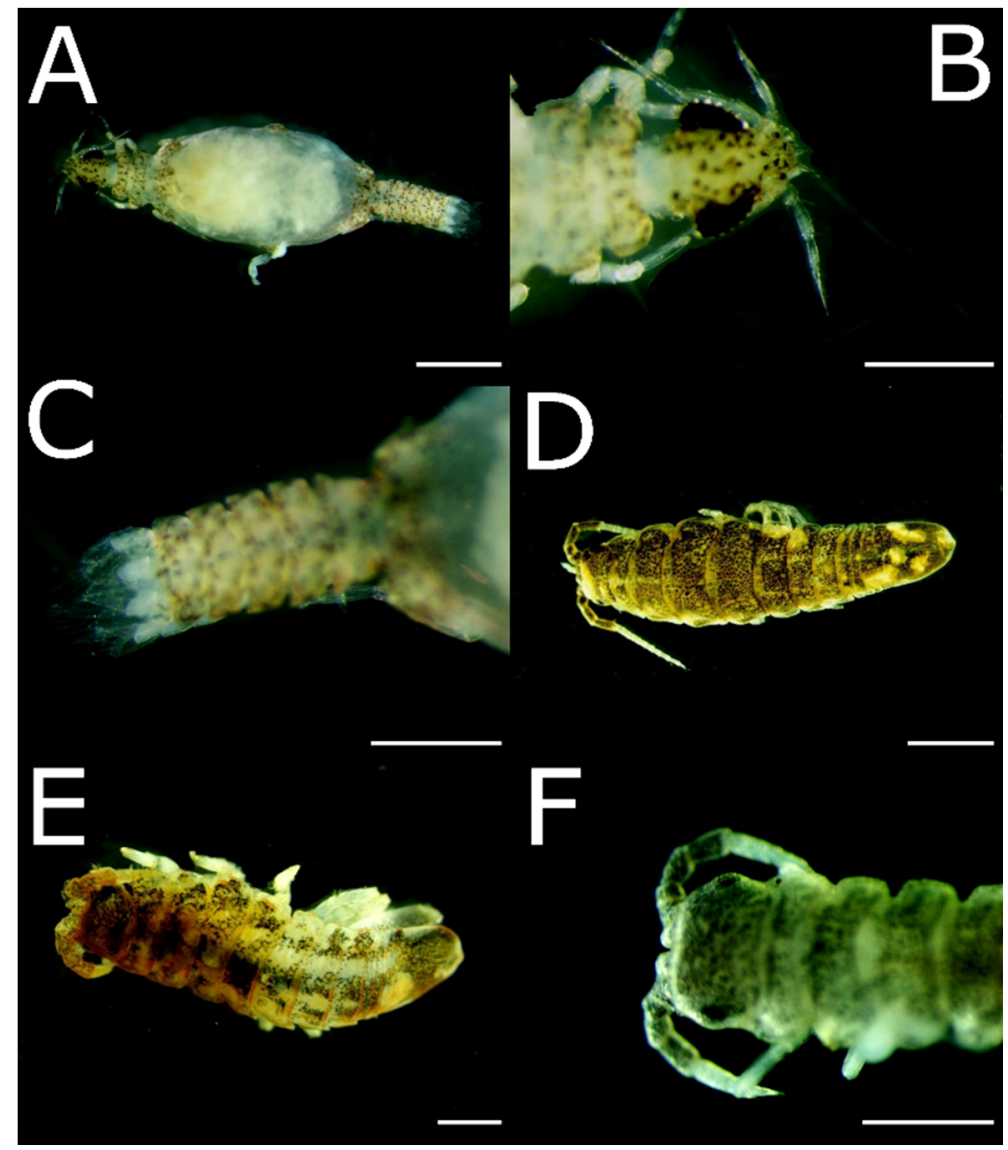

Figure 8. Gnathia vorax (Lucas, 1849): dorsal view (A), cephalon (B), pleotelson (C); Idotea neglecta Sars, 1897: dorsal view (D); Idotea pelagica Leach, 1816: dorsal view (E), and cephalon (F). Scale bars: $(\mathbf{A}, \mathbf{E})=1 \mathrm{~mm} ;(\mathbf{B}, \mathbf{C}, \mathbf{F})=0.5 \mathrm{~mm} ;(\mathbf{D})=2 \mathrm{~mm}$.

Idotea neglecta Sars, 1897 (Figure 8C,D).

Idotea neglecta Sars, 1899: 84-85, Pl. XXXV Fig. 1 [142]; Tattersall, 1911: 225-226, Figs. 106-110 [168]; Naylor, 1955: 491-492, Figs. 1, 2, 7, 8, and 11 [165]; Naylor, 1972: 44, Fig. 12A [6].

Type material: Unknown.

Type locality: Norway [142].

Material examined: One specimen, size $8.79 \mathrm{~mm}$ length, collected the at international Minho River, Caminha $41^{\circ} 52^{\prime} 59^{\prime \prime} \mathrm{N} 8^{\circ} 50^{\prime} 14^{\prime \prime} \mathrm{W}$, May 2020 on glass eel fishing bycatch, deposited as NatMIP-CMIs-0057.

Diagnosis: Body length slightly more than three times the width; antenna 2 flagellum longer than peduncle; antenna 1 reaching the distal end of article 3 of antenna 2 peduncle; pleotelson posterior border laterally rounded with a median obtuse tooth $[6,165]$.

Global distribution: Northeast Atlantic from Norway to France [6].

Distribution in Portugal: Species recorded on Tagus estuary [169] and on Azores archipelago [170].

Ecological notes: Sublittoral on detached algae or fish waste, occasionally on intertidal but also on attached drift algae [6].

Idotea pelagica Leach, 1815 (Figure 8E,F).

Idotea pelagica Leach, 1815a: 365 [171]; Dollfus, 1895: Fig. 23 [172]; Naylor, 1955: 487-489, Figs. 1, 2, 7, 8, and 11 [165]; Naylor, 1972: 44-46, Fig. 13C,F [6]; Hayward \& Ryland, 2017: 345, Fig. 8.24 [22].

Synonyms: Idotea slabberi Bos, 1874: 35 [173].

Idothea pelagica Sars, 1899: 81-82, Pl. XXXIII [142]; Hansen, 1916: 190 [139].

Type material: Unknown. 
Type locality: Scotland [171].

Material examined: Two specimens, size range $2.78 \mathrm{~mm}$ to $6.19 \mathrm{~mm}$ length, collected the International Minho River, Caminha $41^{\circ} 52^{\prime} 04.8^{\prime \prime}$ N $8^{\circ} 51^{\prime} 18.8^{\prime \prime}$ W, June 2021 on beam trawl, deposited as NatMIP-CMIs-0056.

Diagnosis: Antenna shorter than other Idotea species with flagellum shorter than peduncle; telson rounded or with an obtuse median point on adult specimens $[6,165]$.

Global distribution: Northeast Atlantic from Norway to the Iberian Peninsula [6,174].

Distribution in Portugal: Species recorded along the Portuguese west coast [174].

Ecological notes: Common on exposed shores among barnacles and fucoid algae [6].

Suborder Sphaeromatidea Wägele, 1989

Superfamily Sphaeromatoidea Latreille, 1825

Family Sphaeromatidae Latreille, 1825

Genus Cymodoce Leach, 1814

Cymodoce sp. (Figures 9-11).

Material examined: Nine juvenile specimens, size range $2.5 \mathrm{~mm}$ to $3.87 \mathrm{~mm}$, one adult female size $6.88 \mathrm{~m}$ length collected the international Minho River, Caminha $41^{\circ} 52^{\prime} 59^{\prime \prime}$ N 8 $50^{\prime} 14^{\prime \prime}$ W, April 2020 on glass eel fishing bycatch, deposited as NatMIP-CMIs-0063 (juvenile and pereopod 1, pleopod 1, antennule and antenna mounted in blades), NatMIPCMIs-0064 (female and pereopod 1, pleopod 1, antennule and antenna mounted in blades), NatMIP-CMIs-0065 and NatMIP-CMIs-0066; with COI-5P sequence.

Description: Body: Juvenile specimens with uniform color varying bright red to pale greenish yellow (Figure 9A); adult female with orange and yellow bands and molted white spots on pleotelson and most pereonites (Figure 9A,B); head pereon and pleon with a smooth dorsal surface (Figure 9A,B) and pereonite 1 with anterolateral angle extending to the anterior part of the eye on both juvenile and adult female (Figure 9C).

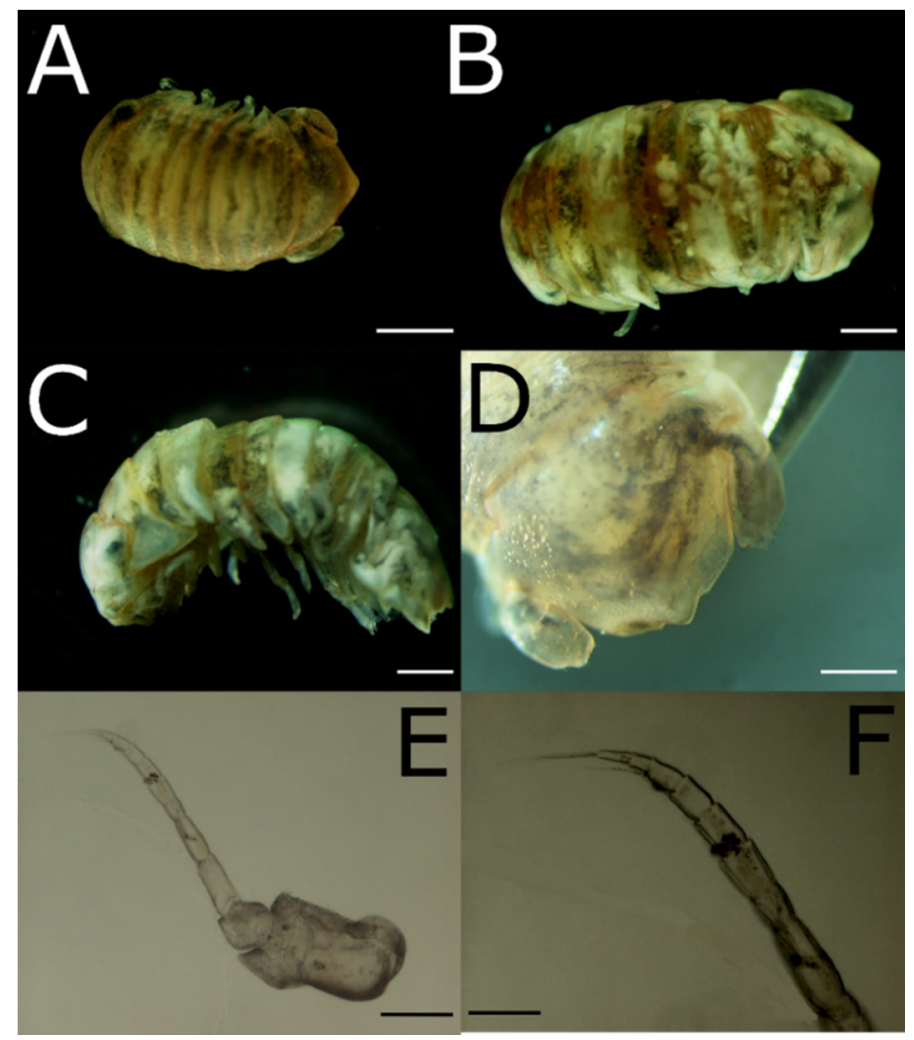

Figure 9. Cymodoce sp.: juvenile dorsal view (A), female adult dorsal view (B), female adult lateral view $(\mathbf{C})$, juvenile pleotelson (D), juvenile antennule (E), and juvenile antennule flagellum (F). Scale bars: $(\mathbf{A}-\mathbf{C})=1 \mathrm{~mm},(\mathbf{D})=0.5 \mathrm{~mm},(\mathbf{E})=0.2 \mathrm{~mm},(\mathbf{F})=0.05 \mathrm{~mm}$. 
Pleotelson: Telson rounded with slightly acute median point (Figure 9D); pleotelson convex on side view (Figure 9C).

Antennule: Juvenile pedunculum with four segments, first segment with an acute corner on inner margin (Figure 9E); flagellum with 7 (Figure 9E) and a large setae on the apex of last segment (Figure 9F); adult female flagellum with 13 segments (Figure 10A,B).

Antenna: Juvenile pedunculum with four segments and flagellum with 10 (Figure 10C); apex of flagellum with three large setae on the apex, posterior end of each segment with a tuff of setae (Figure 10D); adult female pedunculum with four segments and flagellum with 14, posterior end of each segment with a tuff of setae (Figure 10E).

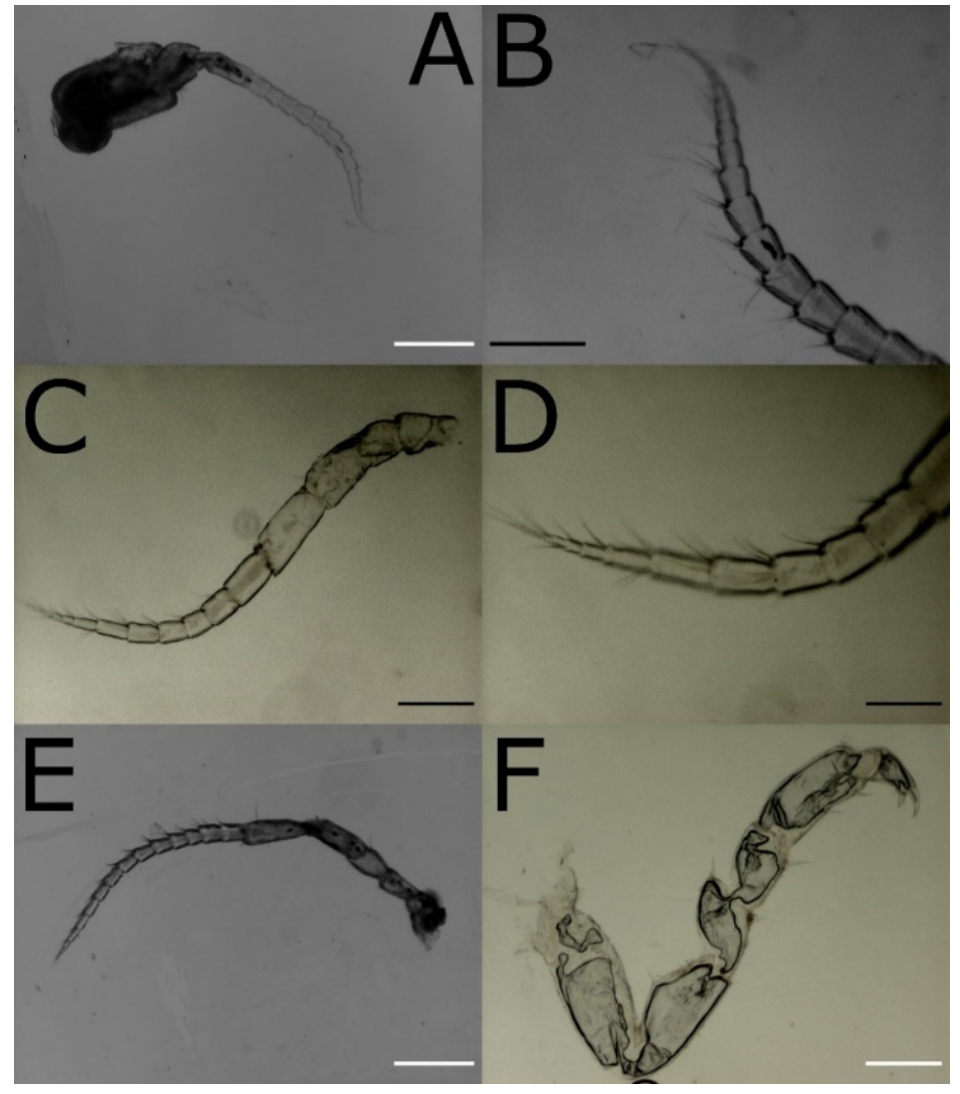

Figure 10. Cymodoce sp.: female antennule (A), female antennule flagellum (B), juvenile antenna (C), juvenile antenna flagellum (D) female antenna (E), and juvenile pereopod 1 (F) Scale bars: $(\mathbf{A}, \mathbf{E})=0.5 \mathrm{~mm} ;(\mathbf{B})=0.25 \mathrm{~mm} ;(\mathbf{C}, \mathbf{F})=0.2 \mathrm{~mm} ;(\mathbf{D})=0.05 \mathrm{~mm}$.

Pereopods: Juveniles with a spine on the middle inner margin of ischium, one spine on each side of the posterior region of merus, one spine on the posterior external margin of carpus, propodus with four spines, one in the middle exterior margin, one on posterior exterior margin and two on posterior inner margin (Figure 10F). Female with four spines on outer margin of carpus (Figure 11A).

Pleopod 1: Juvenile exopod with 19 marginal setae, endopod with 13 on posterior end; anterior margins smooth (Figure 11B), female with smooth basis margins (Figure 11C). 


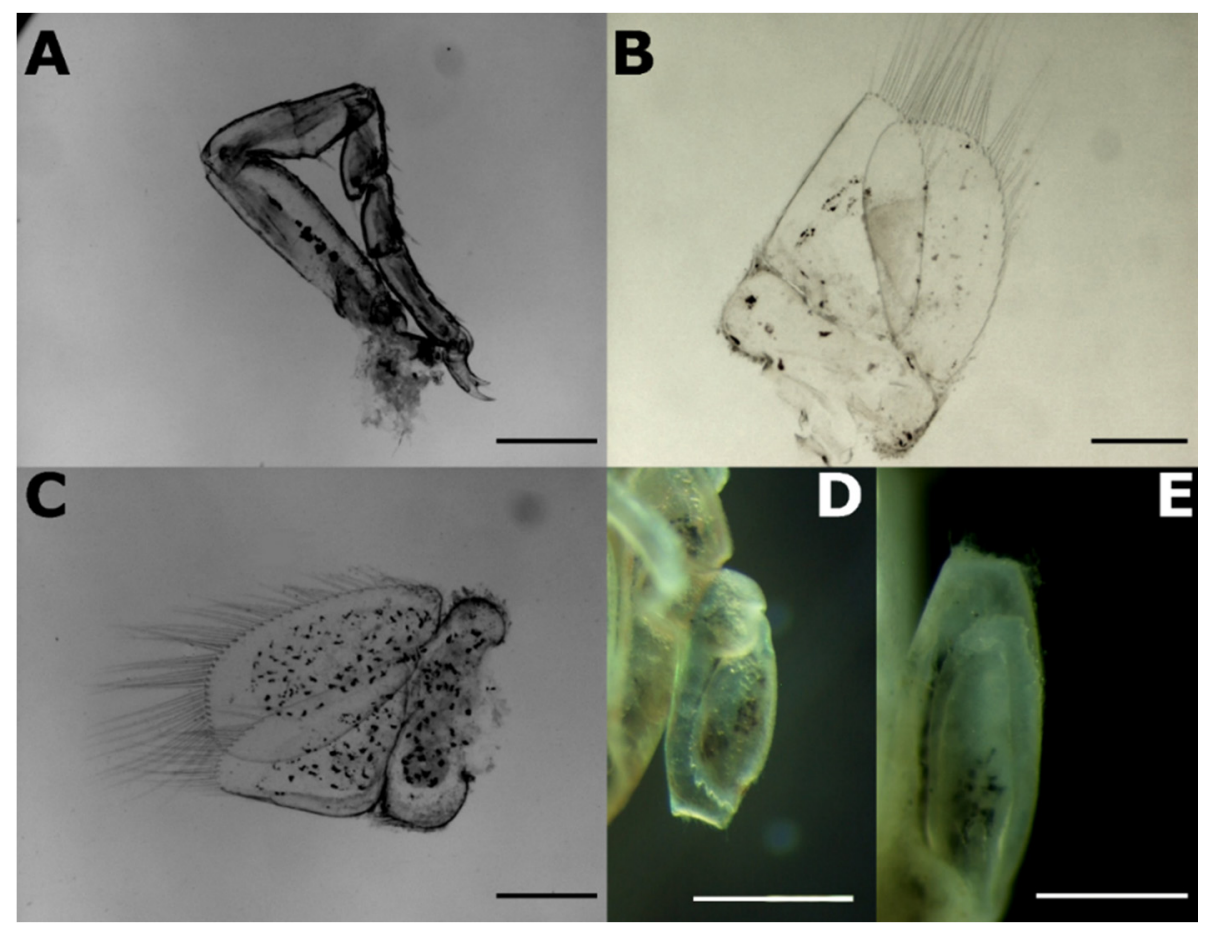

Figure 11. Cymodoce sp.: female pereopod 1 (A), juvenile pleopod 1 (B), female pleopod 1 (C), juvenile uropods (D), and female uropods $(\mathbf{E})$. Scale bars: $(\mathbf{A}, \mathbf{C}-\mathbf{E})=0.5 \mathrm{~mm} ;(\mathbf{B})=0.2 \mathrm{~mm}$.

Uropod: Juveniles uropods slightly smaller than telson; endopod with marked serrations on inner margin; exopod wither than endopod with subquadrate posterior margin and a slightly rounded inner tip (Figure 11D). Female uropods slightly smaller than telson, endopod with crenulated posterior margin, exopod as juveniles with small crenulations on posterior margin (Figure 10E).

Remarks: The genus Cymodoce has six described species for European waters (Atlantic and Mediterranean) [175]. However, antennule, pleopod 1 and uropod shape on both juveniles and female do not seem to match any of the descriptions provided by Dumay [176-179]. The strong acute corner on the inner margin of antennule and pleopod 1 lack of setae and spines on the basis do not match any of the descriptions for females, also juvenile uropod shape has no match with Dumay's descriptions. As most detailed descriptions are for adult males and none male was examined the identification of these specimens is lacking on this aspect, nonetheless the possibility of this record being for a new species cannot be ruled out.

\section{Genetic Analysis}

Both specimens, Ceratothoa aff. oestroides and Cymodoce sp., have not nested on any clade with other publicly available sequences for their respective genera (Figures 12 and 13), nor matched any sequence available on BOLD or GenBank. Ceratothoa aff. oestroides grouped close to the north Pacific species Ceratothoa arimae (Nunomura, 2001), with a sequence divergence of $9.4 \%$, however this branch bootstrap support was 56 . In contrast our sequence did not form a group with other Ceratothoa oestroides sequences that nested within a clade with Ceratothoa italica Schiödte \& Meinert, 1883 with an internal sequence divergence of $0.54 \%$ (Figure 12). Cymodoce sp. grouped closely with a clade formed by sequences of Cymodoce truncata Leach, 1814 and Cymodoce emarginata Leach, 1818 (sequence divergence of $17 \%$ ). However, other sequences of Cymodoce truncata were placed on other branches (Figure 13). 


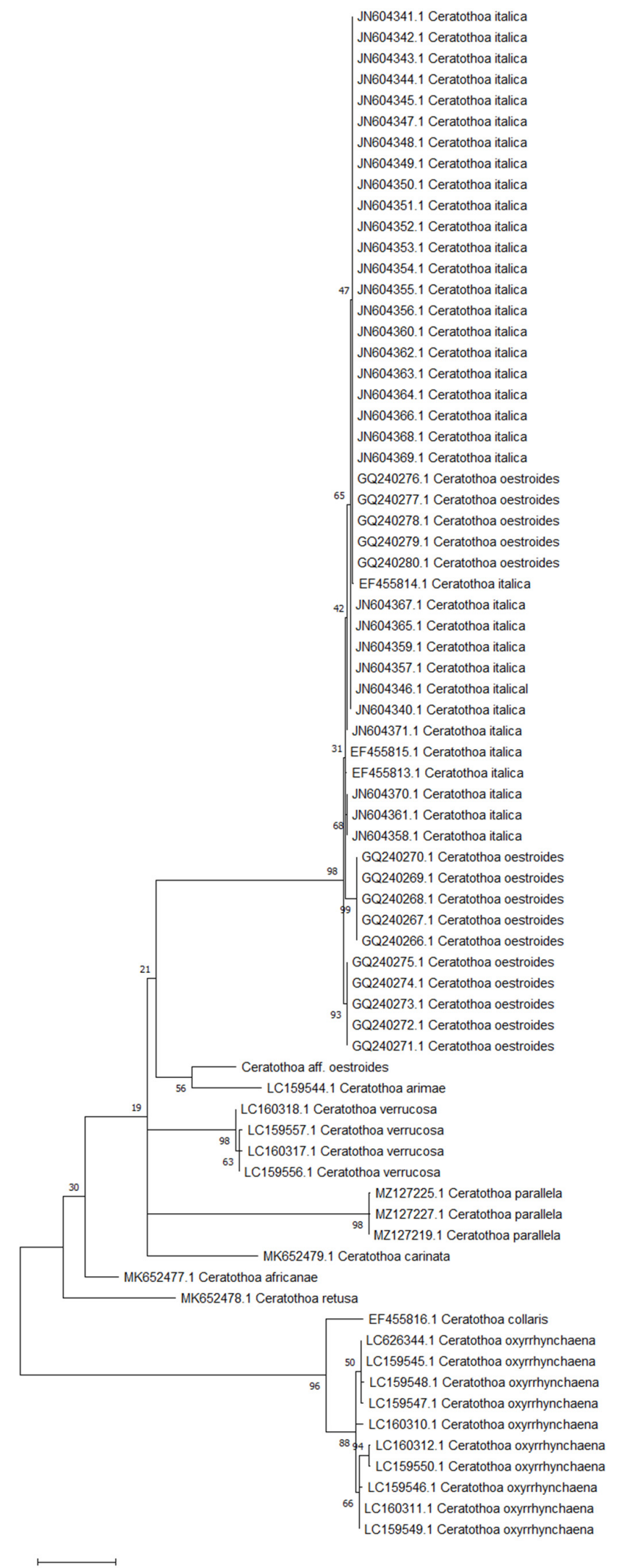

JN604341.1 Ceratothoa italic

N604342.1 Ceratothoa italica

N604343.1 Ceratothoa italica

4.1 Ceratothoa italic

47.1 Ceratothoa italic

604351.1 Ceratothoa italic

2352.1 Ceratothoa ilalica

N604354. 1 Ceratothoa italica

JN604355.1 Ceratothoa italic

04356.1 Ceratothoa italica

GQ240279.1 Ceratothoa oestroides

JN604367.1 Ceratothoa italica

EF455815.1 Ceratothoa italic

GQ240268 1 Ceratothoa oestroides

GQ240267.1 Ceratothoa oestroide

GQ240274.1 Ceratothoa oestroides

GQ240273.1 Ceratothoa oestroides

GQ240272.1 Ceratothoa oestroides

GQ240271.1 Ceratothoa oestroides

0.10

Figure 12. Maximum-likelihood tree obtained from COI-5P sequences of the genus Ceratothoa. Value at nodes corresponds to bootstrap support. 


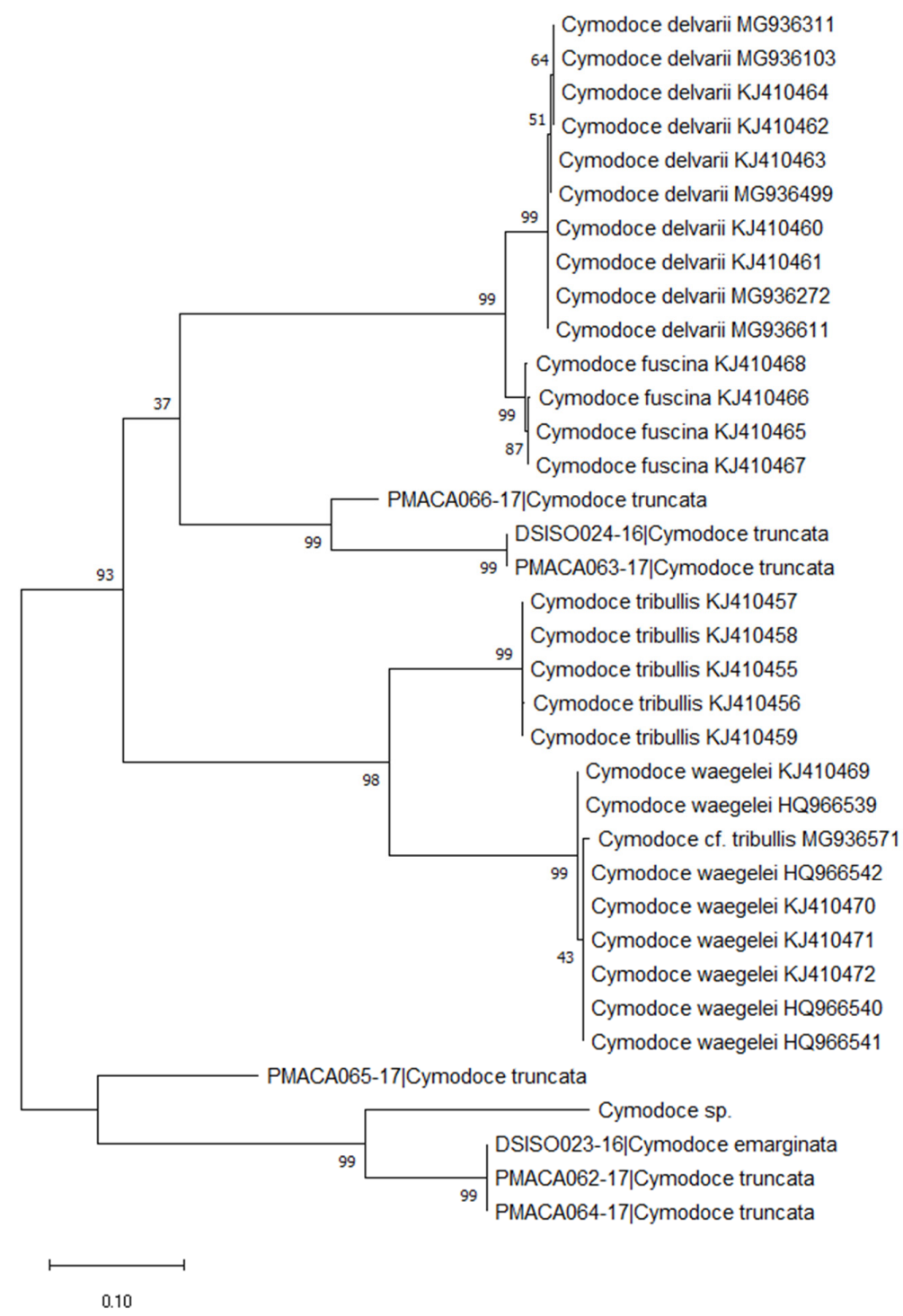

Figure 13. Maximum-likelihood tree obtained from COI-5P sequences of the genus Cymodoce. Value at nodes corresponds to bootstrap support.

\section{Discussion}

From the 23 species collected, six were previously recorded at the Minho River estuary: Procambarus clarkii (Girard, 1852), Carcinus maenas (Linnaeus, 1758), Atyaephyra desmarestii (Millet, 1831), Crangon crangon (Linnaeus, 1758), Gastrosaccus spinifer (Goës, 1864) and Heterotanais oerstedii (Krøyer, 1842) [12,13,15,49], with the remaining 17 species being new records (Table S2). Two of those species are exotic to the Minho River Procambarus clarkia, introduced in the 90s [49], and Austrominius modestus. Decapoda is the most representative group among crustaceans, with freshwater and marine species, while the remaining groups present only marine species on the saltmarsh and near the mouth of the river. Species from the orders Decapoda and Mysida are well documented on the estuary of the Minho River, with four of the six decapod species being already recorded in this area [12,13,49]. Species composition of the order Mysida is similar to assemblages sampled in Ria de Aveiro [113]. Groups like Cumacea, Tanaidacea and Leptostraca are still poorly known in this area.

All species are contained within their documented geographical distribution. Nebalia strausi Risso, 1826 was recorded for the first time on Portuguese waters and Austrominius modestus (Darwin, 1854) on the northern Portuguese coast expanding the known distribution of this species in Portugal. The first record of parasitism from the isopod Ceratothoa oestroides (Risso, 1826) on Alosa alosa (Linnaeus, 1758) is also presented, although confirma- 
tion on C. oestroides identification is still required as COI-5P sequence did not match any of the publicly available sequences for this same species. Although a possible misidentification on the sequences of C. oestroides provided by Mladineo et al., 2009 [180] cannot be ruled out as they nest within a clade formed by sequences of Ceratothoa italica Schiödte \& Meinert, 1883 (internal divergence of $0.54 \%$ ), provided by different groups of authors (Figure 12). As for Cymodoce sp. identification was not possible due to the lack of descriptions and keys for juveniles, being morphologically different from female and male adults, and produced an unmatched COI-5P sequence, requiring a greater effort of adult sampling, identification and posterior DNA barcode sequencing in order to solve problems with juvenile identification or other problematic species in this genus.

Larval stages from Procambarus clarkii found in December are in concordance with the findings on Sousa et al., 2013 [49] which placed the presence of juveniles on the Minho River from June to December. The presence of ovigerous females and megalopes of Palaemon longirostris also coincides with its known breeding periods (April to August [2]), as well as for the presence of larvae and juveniles of Carcinus maenas (starting on March [25]).

\section{Conclusions}

This investigation represents the first taxonomic study of some crustacean groups from the international Minho River, especially some of the less known groups like Cumacea, Tanaidacea and Leptostraca contributing to the knowledge of the Portuguese and Iberian fauna. The species Ceratothoa aff. oestroides was found for the first time parasitizing Alosa alosa. Nebalia strausi had its first record on Portuguese waters. For the genus Cymodoce morphological and molecular approaches are recommended in order to solve its problematic species status and assign juveniles and females to the respective male descriptions as those are still lacking. Despite the number of sampling methodologies employed glass eel fishing bycatch seems to be the most efficient method for sampling marine adventitious crustacean fauna, with the possibility of the appearance of other marine species if the sampling efforts were greater and regular over time. To fully understand the composition and distribution of crustaceans on the estuary of the international Minho River, different sampling methods are required concerning different habitats and assemblages.

Supplementary Materials: The following are available online at https:/ /www.mdpi.com/article/10 $.3390 /$ hydrobiology1010005/s1, Table S1: Genbank accession numbers and BOLD sequence ID for sequences used in genetic analysis; Table S2: Examined species list ( ${ }^{*}$ new records for the Minho river, NW Iberian Peninsula).

Author Contributions: Conceptualization, N.G. and D.A.C.; methodology, N.G.; software, N.G.; validation, N.G.; formal analysis, N.G.; investigation, N.G.; resources, C.A.; data curation, D.A.C., H.C. and C.A.; writing-original draft preparation, N.G.; writing—review \& editing, D.A.C., H.C. and C.A.; visualization, N.G., D.A.C., H.C. and C.A.; supervision, C.A. All authors have read and agreed to the published version of the manuscript.

Funding: This research received no external funding.

Institutional Review Board Statement: Not applicable.

Informed Consent Statement: Not applicable.

Data Availability Statement: Supporting data found at GenBank with the following accession numbers Ceratothoa aff. oestroides OK642789 and Cymodoce sp. OK646549.

Acknowledgments: We thank the facilities provided by "Aquamuseu do Rio Minho", V.N. Cerveira, Portugal. Special thanks to Juan Moreira, Johann Wolfgang Wägele and Kerry Hadfield for their assistance with identification of Leptostraca, Cymodoce and Ceratothoa species respectively.

Conflicts of Interest: The authors declare no conflict of interest. 


\section{References}

1. Ahyong, S.T.; Lowry, J.K.; Alonso, M.; Bamber, R.N.; Boxshall, G.A.; Castro, P.; Gerken, S.; Karaman, G.S.; Goy, J.W.; Jones, D.S.; et al. Animal biodiversity: An outline of higher-level classification and survey of taxonomic richness. Zootaxa 2011, 3148, 165-191. [CrossRef]

2. Smaldon, G. Coastal Shrimps and Prawns; Barnes, R.S.K., Crothers, J.H., Eds.; The Linnean Society of London \& The Estuarine and Brackish-Water Sciences Association: Shrewsbury, UK, 1993.

3. Souza, A.T.; Dias, E.; Marques, J.C.; Antunes, C.; Martins, I. Population structure, production and feeding habit of the sand goby Pomatoschistus minutus (Actinopterygii: Gobiidae) in the Minho estuary (NW Iberian Peninsula). Environ. Biol. Fishes 2015, 98, 287-300. [CrossRef]

4. Mota, M.; Antunes, C. A preliminary characterisation of the habitat use and feeding of Allis shad (Alosa alosa) juveniles in the Minho River tidal freshwater wetlands. Limnetica 2012, 31, 165-172.

5. Lages, A.; Costa, D.D.A.; Gomes, N.; Antunes, C. Exotic Pumpkinseed Sunfish Lepomis gibbosus (Linnaeus, 1758) in the International Minho River (Iberian Peninsula), and Parasitic Association with Myzobdella lugubris Leidy, 1851 (Annelida, Hirudinea). Oceanogr. Fish. Open Access J. 2021, 13, 1-14. [CrossRef]

6. Naylor, E. British Marine Isopods: Keys and Notes for the Identification of the Species, 2nd ed.; Academic Press: London, UK, 1972; ISBN 0125151500

7. Poore, A.G.B.; Campbell, A.H.; Steinberg, P.D. Natural densities of mesograzers fail to limit growth of macroalgae or their epiphytes in a temperate algal bed. J. Ecol. 2009, 97, 164-175. [CrossRef]

8. Dalei, J.; Sahoo, D. Extraction and characterization of Astaxanthin from the crustacean shell waste from shrimp processing industries. Int. J. Pharm. Sci. Res. 2015, 6, 2532-2537. [CrossRef]

9. Horton, T. Ceratothoa steindachneri (Isopoda: Cymothoidae) new to British waters with a key to north-east Atlantic and Mediterranean Ceratothoa. J. Mar. Biol. Assoc. UK 2000, 80, 1041-1052. [CrossRef]

10. Dos Santos, A.; González-Gordillo, J.I. Illustrated keys for the identification of the Pleocyemata (Crustacea: Decapoda) zoeal stages, from the coastal region of south-western Europe. J. Mar. Biol. Assoc. UK 2004, 84, 205-227. [CrossRef]

11. Costanza, R.; D'Arge, R.; De Groot, R.; Farber, S.; Grasso, M.; Hannon, B.; Limburg, K.; Naeem, S.; O'Neill, R.V.; Paruelo, J.; et al The value of the world's ecosystem services and natural capital. Nature 1997, 387, 253-260. [CrossRef]

12. Mazé, R.A.; Lastra, M.; Mora, J. Macrozoobentos del estuario del Mino (NO de Espana). Publ. Espec. Inst. Español. Ocean. 1993, 11, 283-290.

13. Sousa, R.; Dias, S.; Freitas, V.; Antunes, C. Subtidal macrozoobenthic assemblages along the River Minho estuarine gradient (north-west Iberian Peninsula). Aquat. Conserv. Mar. Freshw. Ecosyst. 2008, 18, 1063-1077. [CrossRef]

14. Antunes, C.; Weber, M. The glass eel fishery and the by-catch in the Rio Minho after one decade (1981-1982 and 1991-1992). Arch. Pol. Fish. 1996, 4, 131-139.

15. Picanço, T.C.; Almeida, C.M.R.; Antunes, C.; Reis, P.A. Influence of the abiotic characteristics of sediments on the macrobenthic community structure of the Minho estuary saltmarsh (Portugal). Limnetica 2014, 33, 73-88.

16. Gomes, N.M.A.; Costa, D.D.A.; Cantallo, H.C.; Ribeiro, T.J.A.; Antunes, C. Isopods (Crustacea, Malacostraca) from International Minho River, Iberian Peninsula. Oceanogr. Fish. Open Access J. 2021, 13, 555866. [CrossRef]

17. APA. Plano de Gestão de Região Hidrográfica-Região Hidrográfica do Minho e Lima (Rh1); APA: Lisboa, Portugal, 2016.

18. Dias, E.; Morais, P.; Antunes, C.; Hoffman, J.C. Linking terrestrial and benthic estuarine ecosystems: Organic matter sources supporting the high secondary production of a non-indigenous bivalve. Biol. Invasions 2014, 16, 2163-2179. [CrossRef]

19. BirdLife International Important Bird Areas Factsheet: Minho and Coura Estuaries. Available online: http://www.birdlife.org (accessed on 11 March 2021).

20. Sousa, R.; Guilhermino, L.; Antunes, C. Molluscan fauna in the freshwater tidal area of the River Minho estuary, NW of Iberian Peninsula. Ann. Limnol. 2005, 41, 141-147. [CrossRef]

21. Mota, M.; Bio, A.; Bao, M.; Pascual, S.; Rochard, E.; Antunes, C. New insights into biology and ecology of the Minho River Allis shad (Alosa alosa L.): Contribution to the conservation of one of the last European shad populations. Rev. Fish Biol. Fish. 2015, 25, 395-412. [CrossRef]

22. Hayward, P.J.; Ryland, J.S. Handbook of the Marine Fauna of North-West Europe, 2nd ed.; Hayward, P.J., Ryland, J.S., Eds.; Oxford University Press: New York, NY, USA, 2017; ISBN 9780199549443

23. Jones, N.S. British Cumaceans. Arthropoda: Crustacea. Keys and Notes for the Identification of the Species. Synopses of the British Fauna No. 7; The Linnean Society of London: London, UK, 1976.

24. Barnes, R.S.K. The Brackish-Water Fauna of Northeastern Europe; Cambridge University Press: Cambridge, UK, 1994.

25. Zariquiey-Alvarez, R. Crustáceos decápodos ibéricos. Investig. Pesq. Barc. 1968, 32, XV + 1-510.

26. Anastasiadou, C.; Kitsos, M.S.; Koukouras, A. Redescription of Atyaephyra desmarestii (Millet, 1831) (Decapoda, Caridea, Atyidae) based on topotypical specimens. Crustaceana 2006, 79, 1195-1207. [CrossRef]

27. Ledoyer, M. Sur quelques espèces nouvelles d'Iphinoe (Crustacea Cumacea). Discussion et description comparative des espèces européennes déjà connues. Recl. Traveaux Stn. Mar. d'Endoume 1965, 39, 253-294.

28. Mazziotti, C.; Lezzi, M. The cumacean genus Iphinoe (Crustacea: Peracarida) from Italian waters and I. Daphne N. Sp. From the northwestern Adriatic Sea, Mediterranean. Zootaxa 2020, 4766, 331-357. [CrossRef] 
29. Moreira, J.; Díaz-Agras, G.; Candás, M.; Señarís, M.P.; Urgorri, V. Leptostracans (Crustacea: Phyllocarida) from the Ría de Ferrol (Galicia, NW Iberian Peninsula), with description of a new species of Nebalia Leach, 1814. Sci. Mar. 2009, 73, 269-285. [CrossRef]

30. Makings, P. A guide to the British coastal Mysidacea. Field Stud. Counc. 1977, 4, 575-595.

31. Zimmer, C. Die Nordischen Schizopoden; Brandt, K., Apstein, C., Eds.; Lipsius und Tischler: Kiel und Leipzig, Germany, 1909.

32. WoRMS Editorial Board. World Register of Marine Species; WoRMS and Flanders Marine Institute: Flanders, Belgium, 2021.

33. GBIF. Home Page. Available online: https://www.gbif.org (accessed on 13 February 2020).

34. Lobo, J.; Costa, P.M.; Teixeira, M.A.L.; Ferreira, M.S.G.; Costa, M.H.; Costa, F.O. Enhanced primers for amplification of DNA barcodes from a broad range of marine metazoans. BMC Ecol. 2013, 13, 34. [CrossRef]

35. Hebert, P.D.N.; Ratnasingham, S. The Barcode of Life Data System BOLD. Mol. Ecol. Notes 2007, 7, 355-364. [CrossRef]

36. Altschul, S.F.; Gish, W.; Miller, W.; Myers, E.W.; Lipman, D.J. Basic local alignment search tool. J. Mol. Biol. 1990, 215, 403-410. [CrossRef]

37. Benson, D.A. GenBank. Nucleic Acids Res. 2004, 33, D34-D38. [CrossRef]

38. Girard, C.F. A Revision of the North American Astaci, with Observations on Their Habits and Geographic Distribution. Proc. Acad. Nat. Sci. Phila. 1852, 6, 87-91.

39. Hagen, H.A. Monograph of the North American Astacidae; University Press: Cambridge, UK, 1870.

40. Faxon, W. A Revision of the Astacidæ; Museum of Comparative Zoology, Harvard University: Cambridge, UK, 1885.

41. Campos, E.; Rodríguez-Almaraz, G.A. Distribution of the Red Swamp Crayfish Procambarus clarkii (Girard, 1852) (Decapoda: Cambaridae) in Mexico: An Update. J. Crustac. Biol. 1992, 12, 627-630. [CrossRef]

42. Holdich, D.M.; Reynolds, J.D.; Sibley, P.J. A review of the ever increasing threat to European crayfish from non-indigenous crayfish species. Knowl. Manag. Aquat. Ecosyst. 2009, 11, 394-395. [CrossRef]

43. Wizen, G.; Galil, B.S.; Shlagman, A.; Gasith, A. First record of red swamp crayfish, Procambarus clarkii (Girard, 1852) (Crustacea: Decapoda: Cambaridae) in Israel-Too late to eradicate ? Aquat. Invasions 2008, 3, 181-185. [CrossRef]

44. Harper, D.M.; Smart, A.C.; Coley, S.; Schmitz, S.; Beauregard, G.D.; North, R.; Adams, C.; Obade, P.; Kamau, M. Distribution and abundance of the Louisiana red swamp crayfish Procambarus clarkii Girard at Lake Naivasha, Kenya between 1987 and 1999. Hydrobiologia 2002, 488, 143-151. [CrossRef]

45. Foster, J.; Harper, D.M. Status and Ecosystem Interactions of the Invasive Louisianan Red Swamp Crayfish Procambarus clarkii in East Africa. In Biological Invaders in Inland Waters: Profiles, Distribution, and Threats; Gherardi, F., Ed.; Springer International Publishing: Dordrecht, The Netherlands, 2007; pp. 91-101, ISBN 9781402060298.

46. Magalhães, C.; Bueno, S.; Bond-Buckup, G.; Valenti, W.C.; Silva, H.L.M.; Kiyohara, F.; Mossolin, E.C.; Rocha, S.S. Exotic species of freshwater decapod crustaceans in São Paulo, Brazil: Records and possible the state of Sa causes of their introduction. Biodivers. Conserv. 2005, 14, 1929-1945. [CrossRef]

47. Xu, H.; Qiang, S.; Han, Z.; Guo, J.; Huang, Z.; Sun, H.; He, S.; Ding, H.; Wu, H.; Wan, F. The status and causes of alien species invasion in China. Biodivers. Conserv. 2006, 15, 2893-2904. [CrossRef]

48. Kawai, T. A review of the spread of Procambarus clarkii across Japan and its morphological observations. Freshw. Crayfish 2017, 23, 41-53. [CrossRef]

49. Sousa, R.; Freitas, F.E.P.; Mota, M.; Nogueira, A.J.A.; Antunes, C. Invasive dynamics of the crayfish Procambarus clarkii (Girard, 1852) in the international section of the River Minho (NW of the Iberian Peninsula). Aquat. Conserv. Mar. Freshw. Ecosyst. 2013, 23, 656-666. [CrossRef]

50. Bernardo, J.M.; Costa, A.M.; Bruxelas, S.; Teixeira, A. Dispersal and coexistence of two non-native crayfish species (Pacifastacus leniusculus and Procambarus clarkii ) in NE Portugal over a 10-year period. Knowl. Manag. Aquat. Ecosyst. 2011, 401, 28. [CrossRef]

51. Adao, H.; Marques, J.C. Population Biology of the Red Swamp Crayfish Procambarus Clarkii (Girard, 1852) in Southern Portugal. Crustaceana 1993, 65, 336-345. [CrossRef]

52. Correia, A.M.; Ferreira, O. Burrowing behaviour of the introduced red swamp crayfish Procambarus clarkii (Decapoda: Cambaridae) in Portugal. J. Crustac. Biol. 1995, 15, 248-257. [CrossRef]

53. Aquiloni, L.; Ilhéu, M.; Gherardi, F. Habitat use and dispersal of the invasive crayfish Procambarus clarkii in ephemeral water bodies of Portugal. Mar. Freshw. Behav. Physiol. 2005, 38, 225-236. [CrossRef]

54. Gherardi, F.; Tricarico, E.; Ilhéu, M. Movement patterns of an invasive crayfish, Procambarus clarkii, in a temporary stream of southern portugal. Ethol. Ecol. Evol. 2002, 14, 183-197. [CrossRef]

55. Geiger, W.; Alcorlo, P.; Baltanás, A.; Montes, C. Impact of an introduced Crustacean on the trophic webs of Mediterranean wetlands. Biol. Invasions 2005, 7, 49-73. [CrossRef]

56. Linnaeus, C. Systema Naturae per Regna Tria Naturae, Secundum Classes, Ordines, Genera, Species, Cum Characteribus, Differentiis, Synonymis, Locis; Impensis Direct. Laurentii Salvii: Holmiæ [Stockholm], Sweden, 1758.

57. Herbst, J.F.W. Versuch einer Naturgeschichte der Krabben und Krebse: Nebst einer Systematischen Beschreibung ihrer Verschiedenen Arten; Bei Gottlieb August Lange: Berlin, Germany, 1790.

58. Fabricius, J.C. Mantissa Insectorum Sistens Eorum Species Nuper Detectas Adjectis Characteribus Genericis Differentiis Specificis, Emendationibus, Observationibus. Tome I; Christ. Gottl. Proft: Hafniae [København], Denmark, 1787.

59. Montagu, G. Description of several marine animals found on the south coast of Devonshire. Trans. Linn. Soc. Lond. 1804, 7, 61-85. [CrossRef]

60. Say, T. An account of the Crustacea of the United States. J. Acad. Nat. Sci. Phila. 1817, 1, 97-101. 
61. Leach, W.E. Malacostraca Podophthalmata Britanniae, or; Descriptions of such British Species of the Linnean Genus Cancer as have Their Eyes Elevated on Footstalks; Illustrated with Figures of All the Species by James Sowerby; J. Sowerby: London, UK, 1815.

62. Kinahan, J.R. On Xantho rivulosa and other decapodous Crustacea occurring at Valentia Island, Co. Kerry. Proc. Dublin Nat. Hist. Soc. 1857, 4, 9-16.

63. Ahyong, S.T. Range extension of two invasive crab species in eastern Australia: Carcinus maenas (Linnaeus) and Pyromaia tuberculata (Lockington). Mar. Pollut. Bull. 2005, 50, 460-462. [CrossRef]

64. Carlton, J.T.; Cohen, A.N. Episodic Global Dispersal in Shallow Water Marine Organisms: The Case History of the European Shore Crabs Carcinus maenas and C. aestuarii. J. Biogeogr. 2003, 30, 1809-1820. [CrossRef]

65. Klassen, G.; Locke, A. A Biological Synopsis of the European Green Crab, Carcinus maenas. Can. Manuscr. Rep. Fish. Aquat. Sci. 2007, 2818, 1-82. [CrossRef]

66. Sousa, R.; Dias, S.; Antunes, J.C. Spatial subtidal macrobenthic distribution in relation to abiotic conditions in the Lima estuary, NW of Portugal. Hydrobiologia 2006, 559, 135-148. [CrossRef]

67. Carvalho, A.N.; Santos, P.T. Factors affecting the distribution of epibenthic biodiversity in the Cávado estuary (NW Portugal). Rev. Gestão Costeira Integr. 2013, 13, 101-111. [CrossRef]

68. Amaral, V.; Cabral, H.N.; Jenkins, S.; Hawkins, S.; Paula, J. Comparing quality of estuarine and nearshore intertidal habitats for Carcinus maenas. Estuar. Coast. Shelf Sci. 2009, 83, 219-226. [CrossRef]

69. Anastasiadou, C.; Koukouras, A.; Mavidis, M.; Chartosia, N.; Mostakim, M.; Christodoulou, M.; Aslanoglou, C. Morphological variation in Atyaephyra desmarestii (Millet, 1831) within and among populations over its geographical range. Mediterr. Mar. Sci. 2004, 5, 5-14. [CrossRef]

70. Christodoulou, M.; Antoniou, A.; Magoulas, A.; Koukouras, A. Revision of the freshwater genus Atyaephyra (Crustacea, Decapoda, Atyidae) based on morphological and molecular data. Zookeys 2012, 229, 53-110. [CrossRef] [PubMed]

71. Rafinesque, C.S. Précis des Découvertes et Travaux Somiologiques de m.r C. S. Rafinesque-Schmaltz Entre 1800 et 1814 ou Choix Raisonné de Ses Principales Découvertes en Zoologie et en Botanique, Pour Servir D'introduction à Ses Ouvrages Futurs; Royal Typographie Militaire: Palerme, Italy, 1814.

72. Millet, P.A. Description d'une nouvelle espèce de Crustacé, l'Hippolyte de Desmarets. Mémoires Société D'agriculture Sci. Arts d'Angers 1831, 1, 55-57.

73. Joly, M. Études sur les moeurs, le développement et les métamorphoses d'une petite salicoque d'eau douce (Caridina desmarestii). Ann. Sci. Nat. Zool. 1843, 19, 34-86.

74. De Brito Capelo, F. Descripção de Algumas Especies Novas ou Pouco Conhecidas de Crustaceos e Arachnidios de Portugal e Possessões Portuguezas do Ultramar; Typ. da Academia: Lisboa, Portugal, 1866.

75. Bouvier, E.L. Les variations d'une crevette de la famille des Atyidés, l'Atyaephyra desmaresti Millet. Bull. du Muséum Natl. d'Histoire Nat. 1913, 19, 65-74.

76. Fidalgo, M.L.; Gerhardt, A. Distribution of the freshwater shrimp, Atyaephyra desmarestii (Millet, 1831) in Portugal (Decapoda, Natantia). Crustaceana 2002, 75, 1375-1385. [CrossRef]

77. Fabricius, J.C. Entomologia Systematica emendata et aucta, secundum classes, ordines, genera, species adjectis synonimis locis observationibus descriptionibus. Hafniae: Impensis Christ. Gottl. Proft. 1798, 1-572.

78. Risso, A. Histoire Naturelle des Crustacés des Environs de Nice; Librairie Grecque-Latine-Allemande: Paris, France, 1816.

79. Rathke, H. Zur Fauna der Krym. III; Mémoires de l'Académie Impériale des Sciences de Saint Pétersbourg: Tartu, Estonia, 1837. [CrossRef]

80. Czerniavsky, V. Materialia ad Zoographiam Ponticam Comparatam. Fasc II. Crustacea Decapoda Pontica Littoralia; Universitetskoi Tip: Kharkov, Ukrayne, 1884; Supplement XIII.

81. Campos, J.; Freitas, V.; Pedrosa, C.; Guillot, R.; van der Veer, H.W. Latitudinal variation in growth of Crangon crangon (L.): Does counter-gradient growth compensation occur? J. Sea Res. 2009, 62, 229-237. [CrossRef]

82. Quintaneiro, C.; Monteiro, M.; Pastorinho, R.; Soares, A.M.V.M.; Nogueira, A.J.A.; Morgado, F.; Guilhermino, L. Environmental pollution and natural populations: A biomarkers case study from the Iberian Atlantic coast. Mar. Pollut. Bull. 2006, 52, 1406-1413. [CrossRef]

83. Viegas, I.; Marques, S.C.; Bessa, F.; Primo, A.L.; Martinho, F.; Azeiteiro, U.M.; Pardal, M.Â. Life history strategy of a southern European population of brown shrimp (Crangon crangon L.): Evidence for latitudinal changes in growth phenology and population dynamics. Mar. Biol. 2012, 159, 33-43. [CrossRef]

84. Moreira, F.; Assis, C.A.; Almeida, P.R.; Costa, J.L.; Costa, M.J. Trophic relationships in the community of the upper Tagus estuary (Portugal): A preliminary approach. Estuar. Coast. Shelf Sci. 1992, 34, 617-623. [CrossRef]

85. Luttikhuizen, P.C.; Campos, J.; van Bleijswijk, J.; Peijnenburg, K.T.C.A.; van der Veer, H.W. Phylogeography of the common shrimp, Crangon crangon (L.) across its distribution range. Mol. Phylogenet. Evol. 2008, 46, 1015-1030. [CrossRef]

86. Milne Edwards, H. Histoire Naturelle des Crustacés, Comprenant l'Anatomie, la Physiologie et la Classification de ces Animaux Tome 2; Encyclopédique Roret: Paris, France, 1837.

87. Pennant, T. British Zoology. A New Edition in Four Volumes. Class V. In Crustacea. Class VI. Vermes; Wilkie \& Robinson: London, UK, 1812; Volume IV.

88. Heller, C. Die Crustaceen des Südlichen Europa. Crustacea Podophthalmia. In Mit Einer Übersicht Über Die Horizontale Verbreitung Sämmtlicher Europäischer Arten; Wilhelm Braumüller: Wien, Austria, 1863. 
89. Fischer, P. Crustacés podopthalmaires et cirrhipèdes du Départment de la Gironde. Actes Société Linnéenne Bordx. 1872, 28, 405-438.

90. Cartaxana, A. Distribution and migrations of the prawn Palaemon longirostris in the Mira River estuary (southwest Portugal). Estuaries 1994, 17, 685-694. [CrossRef]

91. Neves, A.; Cabral, H.N.; Gordo, L.S. Distribution and Abundance Patterns of Decapod crustaceans in the Sado estuary, Portugal. Crustaceana 2007, 80, 97-112.

92. França, S.; Pardal, M.A.; Cabral, H.N. Mudflat nekton assemblages in the Tagus estuary (Portugal): Distribution and feeding patterns. Sci. Mar. 2008, 72, 591-602. [CrossRef]

93. Williamson, D.I.; Rochanaburanon, T. A new species of Processidae (Crustacea, decapoda, caridea) and the larvae of the north european species. J. Nat. Hist. 1979, 13, 11-33. [CrossRef]

94. Sampaio, L.; Mamede, R.; Ricardo, F.; Magalhães, L.; Rocha, H.; Martins, R.; Dauvin, J.C.; Rodrigues, A.M.; Quintino, V. Soft-sediment crustacean diversity and distribution along the Portuguese continental shelf. J. Mar. Syst. 2016, 163, 43-60. [CrossRef]

95. Sars, G.O. Middelhavets Cumaceer. Arch. Math. Og Nat. 1878, 3, 461-512.

96. Stebbing, T.R.R. Cumacea (Sympoda); R. Friedländer und Sohn: Berlin, Germany, 1913.

97. Corbera, J.; Garcia-Rubies, A. Cumaceans (Crustacea) of the Medes Islands (Catalonia, Spain) with special attention to the genera Bodotria and Iphinoe. Sci. Mar. 1998, 62, 101-112. [CrossRef]

98. Ferreira, M.A.; Andrade, F. Intertidal communities as indicators of environmental change and their potential use in biomonitoring: The Troia Resort (Portugal), a large-scale tourist development, as a case study. Bol.-Inst. Esp. Oceanogr. 2003, 19, 253-264.

99. Dexter, D.M. Soft bottom invertebrates of the Portuguese benthos. Buletim Inst. Nac. Investig. Pescas 1992, 17, 61-68.

100. Carvalho, S.; Barata, M.; Gaspar, M.B.; Pousão-Ferreira, P.; Cancela da Fonseca, L. Enrichment of aquaculture earthen ponds with Hediste diversicolor: Consequences for benthic dynamics and natural productivity. Aquaculture 2007, 262, 227-236. [CrossRef]

101. Cruz, S.; Gamito, S.; Marques, J.C. Spatial distribution of peracarids in the intertidal zone of the Ria Formosa (Portugal). Crustaceana 2003, 76, 411-431. [CrossRef]

102. Goodsir, H. Description of the genus Cuma, and of two new genera nearly allied to it. Edinb. New Philos. J. 1843, 34, 119-129.

103. Bate, C.S. XLI.-On the British Diastylidæ. Ann. Mag. Nat. Hist. 1856, 17, 449-465. [CrossRef]

104. Reis, C.; Marques, V.; Calvário, J.; Marques, J.C.; Melo, R.; Santos, R. Contribuçao para o estudo dos povoamentos bentónicos (substrato rochoso) da costa ocidental portuguesa. Oecologia Aquat. 1982, 6, 119-145.

105. Norman, A.M. VI.-Crustacea Cumacea of the 'Lightning', 'Porcupine', and 'Valorous' Expeditions. Ann. Mag. Nat. Hist. 1879, 3, 54-73. [CrossRef]

106. Sars, G.O. An Account of the Crustacea of Norway, with Short Descriptions and Figures of All the Species, Cumacea; Bergen Museum: Bergen, NJ, USA, 1900; Volume 3.

107. Boeck, A. Beskrivelse og fremlagde Tegninger af 4 norske Decapoder, undersogte af Overlaege Danielssen og ham. Forhandlinger i Videnskabs-Selskabet i Christiana. Forh. Vidensk. Christ. 1863, 1962-1963, 189-190.

108. Sars, G.O. Om den aberrante Krebsdyrgruppe Cumacea og dens nordiske Arter. Forh. Vidensk. Christ. 1864, 1864-65, 128-208.

109. Norman, A.M. On British Mysidae, a family of Crustacea Schizopoda. Ann. Mag. Nat. Hist. Zool. Bot. Geol. 1892, 10, $242-263$. [CrossRef]

110. Goës, A. Crustacea decapoda podophthalma marina Sueciæ, interpositis speciebus norvegicus aliisque vicinis, enumerat. In Öfversigt af Kongliga Vetenskaps-Akademiens Förhandlingar; Norstedt \& Soner: Stockholm, Sweeden, 1864; Volume 20, pp. 161-180.

111. Sim, G. Stalk-eyed Crustacea of the north-east coast of Scotland. Scotitsh Nat. 1872, 1, 182-190.

112. Stebbing, T.R.R. Gastrosaccus spiniferus Goës, newly described and figured. Ann. Mag. Nat. Hist. Zool. Bot. Geol. 1880, 6, 114-118. [CrossRef]

113. Cunha, M.; Sorbe, J.; Moreira, M. Spatial and seasonal changes of brackish peracaridan assemblages and their relation to some environmental variables in two tidal channels of the Ria de Aveiro (NW Portugal). Mar. Ecol. Prog. Ser. 1999, $190,69-87$. [CrossRef]

114. Tattersall, W.M.; Tattersall, O.S. The British Mysidacea; Ray Society: London, UK, 1951.

115. Leach, W.E. Crustaceology. In The Edinburgh Encyclopaedia; Brewster, D., Ed.; Balfour: Edinburgh, UK, 1814.

116. Thompson, J.V. Zoological Researches and Illustrations; or Natural History of Nondescript or Imperfectly Known Animals, in a Series of Memoirs; King and Ridings: Cork, Ireland, 1829.

117. Czerniavsky, V. Monographia Mysidarum inprimis Imperii Rossici. Fasc. 1, 2. Tr. St.-Peterbg. Obs. Estestvoispyt. 1882, $12,1-170$.

118. Remerie, T.; Vierstraete, A.; Weekers, P.H.H.; Vanfleteren, J.R.; Vanreusel, A. Phylogeography of an estuarine mysid, Neomysis integer (Crustacea, Mysida), along the north-east Atlantic coasts. J. Biogeogr. 2009, 36, 39-54. [CrossRef]

119. Rodrigues, A.M.; Meireles, S.; Pereira, T.; Gama, A.; Quintino, V. Spatial patterns of benthic macroinvertebrates in intertidal areas of a Southern European estuary: The Tagus, Portugal. Hydrobiologia 2006, 555, 99-113. [CrossRef]

120. Sars, G.O. Undersøgelser over Christiania-fjordens Dybvansfauna anstillede paa en i Sommeren 1868 foretagen Zoologisk Reise. Nyt. Mag. Nat. 1869, 16, 305-362.

121. Keeble, F.; Gamble, F.W.X. The colour-physiology of higher crustacea. Philos. Trans. R. Soc. B Biol. Sci. 1904, 196, $295-388$. 
122. Cardoso, P.G.; D'Ambrosio, M.; Marques, S.C.; Azeiteiro, U.M.; Coelho, J.P.; Pereira, E. The effects of mercury on the dynamics of the peracarida community in a temperate coastal lagoon (Ria de Aveiro, Portugal). Mar. Pollut. Bull. 2013, 72, 188-196. [CrossRef] [PubMed]

123. Norman, C.A.M. XXII.-A new Heterotanais and a new Eurydice, genera of Isopoda. Ann. Mag. Nat. Hist. 1906, 17, 167-171. [CrossRef]

124. Holdich, D.M.; Jones, J.A. The distribution and ecology of British shallow-water tanaid crustaceans (Peracarida, tanaidacea). J. Nat. Hist. 1983, 17, 157-183. [CrossRef]

125. Krøyer, H. Nye Arter af Slaegten Tanais. Nat. Tidsskr. Ser. I 1842, 4, 167-188.

126. Müller, F. Tanais rhynchites und balticus, neue Arten aus der Ostsee. Arch. Nat. 1852, 18, 87-90.

127. Risso, A. Histoire Naturelle des Principales Productions de l'Europe Méridionale et Particulièrement de Celles des Environs de Nice et des Alpes Maritimes; Tome V; Chez F.G. Levrault, Libraire: Paris, France, 1826.

128. Dahl, E. Crustacea Leptostraca, principles of taxonomy and a revision of european shelf species. Sarsia 1985, 70, 135-165. [CrossRef]

129. Moreira, J.; Quintas Pérez, P.; Souza Troncoso, J. Sobre la presencia de Nebalia strausi Risso, 1826 (Crustacea, Leptostraca) en la Península Ibérica. Boletín Real Soc. Española Hist. Nat. Sección Biológica 2004, 99, 83-92.

130. Moreira, J.; Abad, L.M.; Riera, R. Presencia de Nebalia strausi Risso, 1826 (Crustacea: Leptostraca) en las Islas Canarias. Rev. Acad. Canar. Ciencias 2009, 21, 99-108.

131. McCormack, E.; Ashelby, C.W.; McGrath, D. A review of the Leptostraca of the British Isles with discussion of the genus Sarsinebalia Dahl. Nauplius 2016, 24, 1-19. [CrossRef]

132. Moreira, J.; Gestoso, L.; Troncoso, J.S. Two new species of Sarsinebalia (Crustacea, Leptostraca) from the Northeast Atlantic, with comments on the genus. Sarsia 2003, 88, 189-209. [CrossRef]

133. Darwin, C. A Monograph on the Sub-Class Cirripedia; The Ray Society: London, UK, 1854.

134. Foster, B.A. The Marine Fauna of New Zealand: Barnacles (Cirripedia: Thoracica). Mem. N. Z. Oceanogr. Inst. 1978, 69, 1-143.

135. Hutton, F.W. List of the New Zealand Cirripedia in the Otago Museum. Trans. Proc. N. Z. Inst. 1879, 11, $285-293$.

136. O'Riordan, R.M.; Ramsay, N.F. The current distribution and abundance of the Australasian barnacle Elminius modestus in Portugal. J. Mar. Biol. Assoc. UK 1999, 79, 937-939. [CrossRef]

137. Wirtz, P.; Araújo, R.; Southward, A.J. Cirripedia of Madeira. Helgol. Mar. Res. 2006, 60, 207-212. [CrossRef]

138. Kussakin, O.G. Morskye I Solonovatovodnye Ravnonogie Rakoobrasnye (Isopoda) Cholodnix I Umerennix Vod Severnogo Polushariya. In [Marine and Brackish Isopods (Isopoda) of Cold and Temperate Waters of the Northern Hemisphere] Podotryad Asellota. Chast 2 Janiridae, Santiidae, 1st ed.; Semeistva Opredeliteli po Faune SSSR, Izdavaemye Zoologicheskim Institutom Academii Nauk SSSR, 152; Nauka: Leningrad, Russia, 1988.

139. Hansen, H.J. Crustacea Malacostraca. III-V. The order Isopoda. In The Danish Ingolf-Expedition; H. Hagerup: Copenhagen, Denmark, 1916; Volume 3, pp. 3-262.

140. Fabricius, O. Fauna Groenlandica, Systematice Sistens Animalia Groenlandiae Occidentalis Hactenus Indagata, Quoad Nomen Specificum, Triviale, Vernaculumque; Synonyma Auctorum Plurium, Descriptionem, Locum, Victum, Generationem, Mores, Usum, Cap-turamque Singuli, Proutd; Ioannis Gottlob Rothe: Hafniae \& Lipsiae, Germany, 1780.

141. Kröyer, H. Grönlands Amfipoder. Det Konigelige Danske Vidensk. Selsk. Nat. Og Math. Afh. Ser. 4 1838, 7, $229-326$.

142. Sars, G.O. An Account of the Crustacea of Norway, 2, Isopoda; Cammermeyer: Christiania, Denmark, 1899.

143. Richardson, H. A monograph on the isopods of North America. Bulletin of the United States. Bull. USA Natl. Mus. 1905, 54, 583-717.

144. Forsman, B. Weitere Studien über die Rassen von Jaera albifrons Leach. Zool. Bidr. Fran Upps. 1949, $27,449-463$.

145. Pereira, S.C.G. Diversidade e Biogeografia de Isópodes Intertidais de Substrato Rochoso na Costa Continental Portuguesa; Universidade do Porto: Porto, Portugal, 2004.

146. Meinert, F.V.A.; Schiødte, J.C. Symbolæ ad Monographiam Cymotharum Crustaceorum Isopodum Familiæ; Scripserunt J. C. Schidte et Fr. Meinert; Typis H. H. Thiele: Hauniæ, Denmark, 1879.

147. Carus, J.V. Prodromus Faunae Mediterraneae, Sive Descriptio Animalium Maris Mediterranei incolarum, Quam Comparata Silva Rerum Quatenus Innotuit, Adiectis Locis et Nominibus Vulgaribus Eorumque Auctoribus; E. Schweizerbart: Stuttgart, Germany, 1885.

148. Barrois, T. Catalogue des Crustacés Marins Recuellis Aux Açores. In Durant les Mois D'août et de Septembre 1887; Le Bigot Frères: Lille, France, 1888.

149. Sociedad Española de Historia Natural. Actas de la Sociedad Española de Historia Natural. Anales de la Sociedad Española de Historia Natural; Real Jardín Botánico: Madrid, Spain, 1890; Volume 19, pp. 1-197.

150. Gourret, P. Les Lemodipodes et les Isopodes du golfe de Marseille. Ann. Musée d'Histoire Nat. Marseille Zool. 1891, 4, 1-44.

151. Koelbel, C. Beitraege zur Kenntnis der Crustaceen der Canarischen Inseln. Ann. Nat. Mus. Wien 1892, 7, $105-116$.

152. Graeffe, E. Ubersicht der fauna des Golfes Von Triest. Arb. Zool. Inst. Univ. Wien Zool. Stn. Triest 1902, $13,33-80$.

153. Brian, A. Noti su alcuni Crostacei parassiti dei pesci del Mediterraneo. Boll. Musei Zool. Anat. Comp. Della R. Univ. Genova 1902, 115, 1-16.

154. Nobre, A. Subsídios para o estudo da fauna marinha do norte Portugal. Ann. Sci. Nat. 1903, 8, 37-94.

155. Nobre, A. Subsídios para o estudo da fauna marinha do sul de Portugal. Ann. Sci. Nat. 1903, 8, 153-160.

156. Trilles, J.P. Les Cymothoidae (Crustacea: Isopoda) du Monde (Prodrome pour une faune). Stud. Mar. 1991, 21, 5-288. 
157. Hadfield, K.A.; Bruce, N.L.; Smit, N.J. Redescription of poorly known species of Ceratothoa Dana, 1852 (Crustacea, Isopoda, Cymothoidae), based on original type material. Zookeys 2016, 592, 39-91. [CrossRef] [PubMed]

158. Milne Edwards, H. Histoire Naturelle des Crustacés: Comprenant L'anatomie, la Physiologie et la Classification de ces Animaux Tome 3; Librairie Encyclopédique de Roret: Roret, Kenya; Paris, France, 1840.

159. Lucas, H. Histoire naturelle des Animaux Articules. Exploration scientifique de l'Algerie pendant les annees 1840, $1841,1842$. Sci. Phys. Zool. 1849, 1, 1-403.

160. Nierstrasz, H.F. Die Isopoden-Sammlung im Natuurhistorischen Reichsmuseum zu Leiden: I. Cymothoidae. Zool. Meded. 1915, 1, 71-108.

161. Trilles, J.P. Les Cymothoidae (Isopoda, Flabellifera) des côtes françaises. (Systématique, faunistique, écologie et répartition géographique) I. Les Ceratothoinae Schiœdte et Meinert, 1883. Bull. Muséum Natl. D'histoire Nat. 1972, 91, 1191-1228.

162. Trilles, J.P.; Raibaut, A. Sur les Cymothoidae (Isopoda, Flabellifera) parasites de poissons marins de Tunisie (2e note). Bull. Muséum Natl. D'histoire Nat. 1973, 114, 273-281.

163. Hadfield, K.A.; Smit, N.J. Review of the global distribution and hosts of the economically important fish parasitic isopod genus Ceratothoa (Isopoda: Cymothoidae), including the description of Ceratothoa springbok n. sp. from South Africa. Int. J. Parasitol. 2020, 50, 899-919. [CrossRef]

164. Naylor, E. Isopoda. Sub-Order: Flabellifera, Family: Gnathidae, Genera: Paragnathia, Gnathia, Family: Cirolanidae, Genus: Eurydice. In Fiches D'identification du Zooplancton; ICES, 78: Copenhagen, Denmark, 1957.

165. Naylor, E. The comparative external morphology and revised taxonomy of the British species of Idotea. J. Mar. Biol. Assoc. UK 1955, 34, 467-493. [CrossRef]

166. Carvalho, S.; Marques, J.C.; Banha, M.M.; Cancela da Fonseca, L. Macrobenthic Crustacea of the Bay of S. Torpes (Portugal). Rev. Biol. 2003, 21, 57-70.

167. Gonzalez, P.; Sanchez, M.I.; Chirivella, J.; Carbonell, E.; Riera, F.; Grau, A. A preliminary study on gill metazoan parasites of Dentex dentex (Pisces: Sparidae) from the western Mediterranean Sea (Balearic Islands). J. Appl. Ichthyol. 2004, $20,276-281$. [CrossRef]

168. Tattersall, W.M. Die Nordischen Isopoden; Lipsius \& Tischer: Kiel, Germany, 1911.

169. Cabral, H.N. Comparative feeding ecology of sympatric Solea solea and S. senegalensis, within the nursery areas of the Tagus estuary, Portugal. J. Fish Biol. 2000, 57, 1550-1562. [CrossRef]

170. Borges, P.A.V.; Costa, A.; Cunha, R.; Gabriel, R.; Gonçalves, V.; Martins, A.; Melo, I.; Parente, M.; Raposeiro, P.; Rodrigues, P.; et al. Listagem dos Organismos Terrestres e Marinhos dos Açores-A List of the Terrestrial and Marine Biota from the Azores; Princípia: Cascais, Portugal, 2010; ISBN 9789898131751.

171. Leach, W.E. A tabular view of the external characters of four classes of animals, which Linné arranged under Insecta; with the distribution of the genera composing three of these classes into orders, \&c. and descriptions of several new genera and species. Trans. Linn. Soc. Lond. 1815, 11, 306-400.

172. Dollfus, A. Les Idoteidae des côtes de France. Feuille Des Jeunes Nat. 1895, 25, 1-5, 17-18, 38-40, 53-56.

173. Bos, J.R. Bijdrage Tot de Kennis van de Crustacea Hedriophthalmata van Nederland en Zijne Kusten/Door J. Ritzema Bos; J. B. Huber: Groningen, The Netherlands, 1874.

174. Pereira, S.G.; Lima, F.P.; Queiroz, N.C.; Ribeiro, P.A.; Santos, A.M. Biogeographic patterns of intertidal macroinvertebrates and their association with macroalgae distribution along the Portuguese coast. Hydrobiologia 2006, 555, 185-192. [CrossRef]

175. Khalaji-Pirbalouty, V.; Bruce, N.L.; Wägele, J.W. The genus Cymodoce Leach, 1814 (Crustacea: Isopoda: Sphaeromatidae) in the Persian Gulf with description of a new species. Zootaxa 2013, 3686, 501. [CrossRef]

176. Dumay, D. Révision systématique du genre Cymodoce (Isopoda Flabellifera). I. Introduction et decription de deux espèces: Cymodoce truncata (Montagu) et C. pilosa Milne Edwards. Téthys 1972, 3, 638-654.

177. Dumay, D. Révision systématique du genre Cymodoce (Isopoda Flabellifera), II. Descriptions de Cymodoce hanseni nov. sp. des cotes Méditerranéenes. Crustaceana 1972, Supplement 3, 198-206.

178. Dumay, D. Révision systématique du genre Cymodoce (Isopoda Flabellifera) 3. Description de C. spinosa Risso et de C. emarginata sensu Torelli. Téthys 1972, 4, 127-144.

179. Dumay, D. Révision systématique du genre Cymodoce (lsopoda Flabellifera) 4. Description de C. tattersalli Torelli, C. rubropunctata (Grube), C. tuberculata Costa. Clef systematique et conclusion générale. Téthys 1972, 4, 457-480.

180. Mladineo, I.; Šegvić, T.; Grubišić, L. Molecular evidence for the lack of transmission of the monogenean Sparicotyle chrysophrii (Monogenea, Polyopisthocotylea) and isopod Ceratothoa oestroides (Crustacea, Cymothoidae) between wild bogue (Boops boops) and cage-reared sea bream (Sparus aurata). Aquaculture 2009, 295, 160-167. [CrossRef] 